\title{
LA BIBLIOFAM: PROGRAMA PSICOEDUCATIVO FAMILIAR PARA EL TRATAMIENTO DEL ADULTO MAYOR CON DIABETES TIPO 2
}

\section{THE BIBLIOFAM: FAMILY PSYCHOEDUCATIONAL PROGRAM FOR THE TREATMENT OF OLDER ADULTS WITH TYPE 2 DIABETES}

\section{Lucía Pérez Sánchez}

Profesora investigadora de tiempo completo en la universidad autónoma de Nayarit, México

Cómo referenciar este artículo/How to reference this article:

Pérez Sánchez, L. (2018). La BIBLIOFAM: programa psicoeducativo familiar para el tratamiento del adulto mayor con diabetes tipo 2. Revista de Psicoterapia, 29(110), 211-239.

\section{Resumen}

El siguiente trabajo tuvo como objetivos implementar un programa psicoeducativo familiar en el tratamiento del adulto mayor (AM) con diabetes tipo 2 (DM2), a través de un diseño metodológico de caso clínico con réplica, con pre y post test, se aplicó a 5 familias, los resultados muestran que hubo modificaciones en los indicadores metabólicos, calidad de vida, autoeficacia - con relación a la línea base inicial-pero no así en la variable de la mejor configuración familiar y desgaste del cuidador, que repercutiera en un cuidado más adecuado del AM, por lo que se requiere en futuras aplicaciones observar su impacto otorgándole un rango de tiempo más extenso en el seguimiento, para la verificación de su eficacia así como para garantizar la consecución del proceso cognitivo en el cual se basa la teoría del aprendizaje significativo de Ausubel.

Palabras claves: Adulto mayor, Diabetes tipo 2, familia, psicoeducación.

\begin{abstract}
The aim of this study was to implement a family psychoeducational program in the treatment of older adults with type 2 diabetes (DM2), through a clinical case method design with replication, with pre and posttest, applied to 5 families, the results show that there were modifications in the metabolic indicators, quality of life, self-efficacy -in relation to the initial baselinebut not in the variable of the best family configuration and the burned out caregiver, that would have repercussions in a care more adequate in the elderly people, so that in future applications it is necessary to observe its impact, granting a longer time range in the monitoring, for the verification of its effectiveness as well as to guarantee the achievement of the cognitive process on which the Significant learning theory of Ausubel.
\end{abstract}

Keywords: Elderly people, Type 2 Diabetes, Family, Psychoeducation

Fecha de recepción v1: 10-02-2018. Fecha de recepción v2: 22-04-2018. Fecha de aceptación: 08-01-2018. Correspondencia sobre este artículo:

E-mail: lucia@systemica.com.mx

Dirección postal: Systemica. Terapia Familiar. Rio Bravo 85 Dpto.1. col. Los Fresnos. México (C) 2018 Revista de Psicoterapia 


\section{Introducción}

La actual sociedad debe enfrentar el reto que plantea el envejecimiento de su población. Se estima que el aumento en la esperanza de vida, en personas con más de 75 años, en el mundo se ha incrementado en una proporción de cinco veces más que la del resto de la población Estudio nacional de salud y envejecimiento en México (ENASEM), 2012. Esto replantea nuevas necesidades que atender; necesidades que implican mayor complejidad, sobre todo en lo concerniente al equilibrio de dos factores:

- Promoción de la autonomía del adulto mayor (AM).

- Brindar protección socio sanitaria en situación de dependencia (Muñoz, García y Durán, 2004).

Por lo que, el sistema sanitario no puede ser el único encargado de brindar atención y servicios al alcance de la población, será necesario que una red más amplia pueda brindar apoyo organizado; siendo la familia un sistema con recursos amplios para el logro de este objetivo.

Al respecto, ya López, Orueta, Gómez-Caro, Sánchez, Carmona y Alonso (2009) destacaban que existen al menos cuatro aspectos relacionados con una potencial influencia sobre las familias para aumentar el tiempo dedicado al cuidado de enfermos en el hogar: envejecimiento de la población como principal causa, en segundo lugar, el aumento de padecimientos crónicos, la incorporación de la mujer en la vida laboral, y finalmente los cambios en los sistemas de salud.

Por lo que hace necesario entonces voltear a ver a la familia y proporcionar herramientas que potencialicen sus propios recursos, razón por lo cual la psicoeducación es una vía adecuada para el logro de ello. El siguiente trabajo presenta los resultados de un programa de esta índole. Basado en la teoría del aprendizaje significativo de Ausubel, se planteó a través de la biblioterapia, para el trabajo familiar, a continuación, se muestra los resultados que se obtuvieron.

\section{Objetivo}

Implementar un tratamiento psicoeducativo basado en la biblioterapia denominado BIBLIOFAM, para mejorar la calidad de vida y autoeficacia del AM, regular la sobrecarga del $\mathrm{CP}$, así como el desarrollo de una configuración familiar más funcional que repercuta en el cuidado y atención del AM.

\section{Método}

Con un diseño de estudio de caso único con replica o N=1 (León y Montero, 2002).

\section{Participantes}

Los participantes fueron cinco familias que recibieron el tratamiento de corte psicoeducativo, basado en la biblioterapia, el cual consistió en información bibliográfica que previamente fue seleccionada como relevante para los participantes. 
Criterios de inclusión:

a) Familiar mayor (60 años o más) con padecimiento de diabetes y que está bajo el cuidado de la familia.

b) Pareja del AM o en su defecto el familiar identificado por la propia familia como el que lleva la responsabilidad del cuidado y atención del AM con DM2.

c) Dos familiares que fueron designados por la propia familia y que no hubieran sido previamente identificados como $\mathrm{CP}$.

\section{Tipo de muestreo}

El tipo de muestro no probabilístico por conveniencia, y se utilizó el procedimiento de bola de nieve.

\section{Instrumentos}

Ficha de identificación (diseñado específicamente para esta investigación). Este formato sirvió para la obtener información general de la familia participante tal como: dirección, teléfono, horarios para realizar la intervención.

Cuestionario de preselección (diseñado específicamente para esta investigación). Recogió información general del AM y su familia sobre aspectos del padecimiento. Consistió en cinco preguntas en las cuales se contestaba con Sí o No. Si la familia participante respondía a todas que Sí, se consideraba candidata a participar en la intervención; con excepción de la pregunta número cuatro. La pregunta cuatro tenía que ver con algún impedimento por parte del AM para poder participar, si este era el caso se refirió a otro nivel de atención.

Entrevista de Carga del Cuidador Zarit (ECCZ, Montorio, Izal, López y Sánchez, 1998). La aplicación de la ECCZ, tuvo como objetivo medir la carga percibida por el propio cuidador. Este instrumento consta de 22 ítems cuya opción de respuesta es a través una escala Likert que va de 0 (nunca) a 4 (siempre). El entrevistado debe señalar la frecuencia con la que se siente identificado con las afirmaciones. Las dimensiones que evalúa son: Impacto del cuidado, Relación interpersonal cuidador paciente y Expectativas de autoeficacia.

De acuerdo con el estudio realizado por González-Celis (2009), su validación se llevó a cabo al analizar la estructura del instrumento por medio de un análisis factorial forzado a tres factores, lo que dio como resultado que se obtuviera un á $=.94$, para el primer factor Impacto del cuidado; á=.91 para el segundo Relación cuidador paciente; y á $=.67$ para el tercero Expectativas de autoeficacia. Esta herramienta presenta un coeficiente de correlación (CCI) .71- .85; tiene un alfa de Cronbach de .93; y una $\mathrm{r}$ de Pearson de .71.

Evaluación de Calidad de vida en población de adultos mayores en México (WHOQOL, González - Celis, 2010). La evaluación de calidad de vida del AM se realizó con el objetivo de conocer sus facetas individuales o dimensiones de: Salud física, Independencia, Salud psicológica, Relaciones Sociales, Medio ambiente y 
Espiritualidad. Cada dimensión consta de diez ítems. El instrumento aplicado tiene una validez concurrente, con la correlación entre la medida total de CV arrojada por el WHOQOL-100 y el bienestar general subjetivo de adultos mayores obtenido con la escala de bienestar subjetivo (PGC), siendo de $\mathrm{r}=.87(\mathrm{p}<.0001)$.

También se examinó la validez concurrente a través de la asociación entre CV y la actitud hacia su propio envejecimiento en los adultos mayores, donde existió una correlación positiva entre ambos $(\mathrm{r}=.400 ; p<.0001)$, teniendo con ello que la actitud positiva hacia el envejecimiento se asoció con una mejor $\mathrm{CV}$; por ello este instrumento se aplicó al AM.

Autoeficacia para Realizar Actividades Cotidianas en Ancianos Mexicanos (AeRAC González-Celis, 2009). La medición de la autoeficacia para la realización de actividades cotidianas del AM tiene como objetivo evaluar las Actividades Recreativas, Actividades Sociales y Actividades de Protección de la salud de los AM.

Este instrumento contempla las dimensiones de Actividad de Independencia, Actividades Recreativas Pasivas, Actividades Recreativas Activas, Actividades Sociales, Actividades de Protección a la salud, con un total de 24 ítems. Las opciones de respuesta están dadas por una escala tipo Likert y van desde completamente, con una puntuación de 100, a nada, con una puntuación de 0 . Tiene un alfa de Cronbach de .8454 y un coeficiente de correlación $r$ de Pearson de .612. Este instrumento se aplicó al AM.

Escala de Funcionamiento Familiar (EFF García, Rivera, Reyes-Lagunes, Díaz-Loving, 2006). Este instrumento tuvo como objetivo evaluar las interacciones, la organización y la forma en que funcionan las familias, donde la acción y la reacción de un individuo propician movimientos en los demás integrantes.

Las dimensiones que contempla esta escala son Ambiente Familiar Positivo, Cohesión/ Reglas Familiares, Hostilidad/Evitación del Conflicto, Mando/Problemas en la Expresión de Sentimientos. Con un total de 45 ítems, con base en una escala tipo Likert y van desde: nunca, con una puntuación de 1 y siempre con una puntuación de 5.

Su validación se da en la agrupación de los cuatro factores que explican el 56\% de la varianza total, con una alpha de Cronbach de .8533 . Este instrumento fue contestado en consenso por todos los participantes.

La Bitácora de Lectura. Las bitácoras de lectura tuvieron el propósito de plasmar las inquietudes, reflexiones, comentarios y dudas que surgían con relación a la lectura revisada por cada tema.

\section{Procedimiento}

Las etapas del procedimiento se muestran a continuación. 


\section{1.- Entrenamiento de las terapeutas.}

Se llevó a cabo de manera separada, con la finalidad de llevar a cabo el procedimiento metodológico del doble ciego. es decir, cuando ni el experimentador ni los sujetos conocen las condiciones que sustentan al estudio, y que se emplea como un procedimiento para controlar la variable extraña que podría ser introducida por el investigador y así evitar la amenaza a la validez externa de la investigación.

Las sesiones de entrenamiento tuvieron como objetivo: dar a conocer la perspectiva psicogerontológica del envejecimiento, como proceso complejo, así como la necesidad de aprender a ver al AM como un ser capaz de realizar todo lo que sus capacidades le permitan, con una visión potenciadora vs. asistencial; tendiente al envejecimiento saludable. Además de esta sensibilización por parte de los terapeutas, también este entrenamiento fue necesario para dar a conocer el material de trabajo y las lecturas que se utilizarían. A través de las siguientes temáticas:

- Campo de la Psicogerontología.

- Envejecimiento normal vs. Envejecimiento Patológico.

- Familia y Abuelidad.

- Familia y Enfermedad.

- La Bibliofam (estructura y manual de tratamiento).

\section{2.- Autorización de la institución.}

- Se presentó el protocolo de investigación.

- Se ofrecieron los tratamientos a las familias derechohabientes. De manera gratuita, se hizo el compromiso de compartir la información que arrojará dicho estudio, así como de capacitar al personal a cargo de los AM con DM2 y sus familias.

- El Instituto de Seguridad y Servicios Sociales de los Trabajadores del Estado (ISSSTE) (institución donde se llevó a cabo el estudio) solicitó el protocolo completo de la investigación para ser evaluado por el comité de bioética, el cual autorizó que se llevara a cabo la investigación.

Finalmente, las familias fueron invitadas a través de la médico gerontóloga del ISSSTE, después de concertar la cita, de manera aleatoria (a través de una tómbola) se asignaba a la familia a un tipo de tratamiento. El lugar donde se realizaron las sesiones de la BIBLIOFAM fue también en la cámara Gesell del Programa Académico de Psicología de la Universidad Autónoma de Nayarit. A continuación, se describe la aplicación de este tratamiento.

\section{3.- Aplicación de la BIBLIOFAM.}

El procedimiento de construcción del diseño de la BIBLIOFAM surgió a partir de la necesidad para implementarse en el contexto familiar; por lo que se decidió por la Biblioterapia como opción para el desarrollo del segundo tratamiento. $\mathrm{Su}$ fundamento es la lectura dirigida y la discusión grupal (familia), favoreciendo la 
interacción entre los participantes; facilitando la expresión de sentimientos, miedos, angustias y ansiedades que la misma lectura evoca; además de que propiciaba el intercambio de experiencias y valores pues conduce a la reflexión; y por su enfoque educativo ayuda a la adquisición de nuevos hábitos y mejora los comportamientos y formas de comunicación (Gold, 2008). La BIBLIOFAM consistió en tres etapas:

Cada etapa tuvo sesiones estructuradas y con actividades definidas para la consecución de metas específicas.

Primer etapa evaluación: recolección de la información referente a datos generales y socioeconómicos de la familia. Aplicación de los instrumentos para llevar a cabo el pre test para realizar la medición de los indicadores metabólicos, la autoeficacia y la calidad de vida del AM, la sobrecarga del CP, y la configuración familiar.

Segunda etapa intervención: establecer cambios para el desarrollo de una configuración familiar más funcional. Analizar la manera que como familia tienen para afrontar y resolver las distintas dificultades familiares. Conocer y de ser necesario modificar el tipo de vinculación familiar para mejorar el cuidado y atención del AM. Establecer formas comunicativas más funcionales que impacten de manera adecuada en el cuidado y atención del AM. Modificar los patrones de comportamiento no saludables que pueden potenciar el deterioro por la enfermedad crónica.

Cada sesión contempló actividades que generarán la construcción de aprendizajes significativos, organizadas de forma consecutiva de acuerdo con el proceso cognitivo, de tal manera que se recuperaba los aprendizajes previos de cada participante en relación a la temática que se abordaba, para, posteriormente, pasar al proceso de articulación y construcción significativa de lo aprendido en un proceso integrativo (Novak y Gowin, 1988):

- Actividad preliminar: el objetivo era problematizar a los participantes en relación con el tema que se revisaría en esa sesión. Esto a través de una lectura previa de la temática y con diversas estrategias.

- Actividad de aprendizaje: el objetivo fue que los participantes pudieran construir en colaboración, el aprendizaje que lograron en la revisión de la temática de la sesión.

- Actividad integradora: el objetivo consistió en que todos los participantes explicaran cómo construyeron el aprendizaje de la temática que se revisó en sesión, de manera clara y concreta, a través de recursos lúdicos y creativos.

La esencia de la BIBLIOFAM se basó en el análisis en círculo de lectura familiar; es decir, el objetivo fue intercambiar opiniones sobre las lecturas, o revisión de películas que la familia participante leyó en casa, a la luz de sus propias experiencias. Por ello, para su óptimo trabajo, debían realizar una Bitácora de Lectura, en la cual se plasmaba las impresiones y reflexiones que tenía cada uno de los participantes, y que servía para la discusión y análisis de lo aprendido en sesión. 
Tercera etapa seguimiento: se realizó a los tres meses de finalizado el tratamiento; se evaluaron los indicadores metabólicos del AM, y se aplicaron los instrumentos de medición de las otras variables: autoeficacia, calidad de vida, desgaste del cuidador y funcionamiento familiar.

\section{Analisis de datos}

Basados en el diseño de caso único con replica, se establecio la linea base previa a la intervención, posteriormente se repitió la medición terminado el tratamiento; para la interpretación se recurrió a una apreciación no estadística, observando la evolución del tratamiento en tablas y gráficas, las cuales registran un número de series, y se observan los cambios ocurridos a la linea base (León y Montero, 2002).

\section{Resultados y Discusión}

Los resultados en cuanto a las modificaciones que sufrieron las mediciones de los indicadores metabólicos muestran que hubo cambios posterior a la intervención, pero durante el seguimiento regresaron a las mediciones de la línea base (ver Tabla 1), en tres de los participantes los cambios fueron moderados en los indicadores de colesterol y triglicéridos, en otro participante en la hemoglobina glucosada y uno más lo mostró en la glucosa, pero los cinco tuvieron mejorías en las mediciones de su presión arterial; coincidiendo con lo que describen Calderon, Solis, Castillo, Cornejo, Figueroa, Paredes, Manrique y Neyra, (2003) y Norris, Lau, Smith, Schmid y Engelgau (2002) sobre los efectos de intervenciones educativas en el control metabólico en pacientes con diabetes. Sus hallazgos demuestran que posterior a los tratamientos hubo un mejor control, pero posterior a los seis meses de seguimiento nuevamente regresaron a las mediciones del pre test, sin embargo, existió un mayor porcentaje de controlar nuevamente la Hemoglobina glucosilada (HbA1c), que los pacientes que no tuvieron este tipo de intervenciones (Fernández, Abdala, Alvara, Tenorio, López, Cruz, Dávila, y González, 2012). 


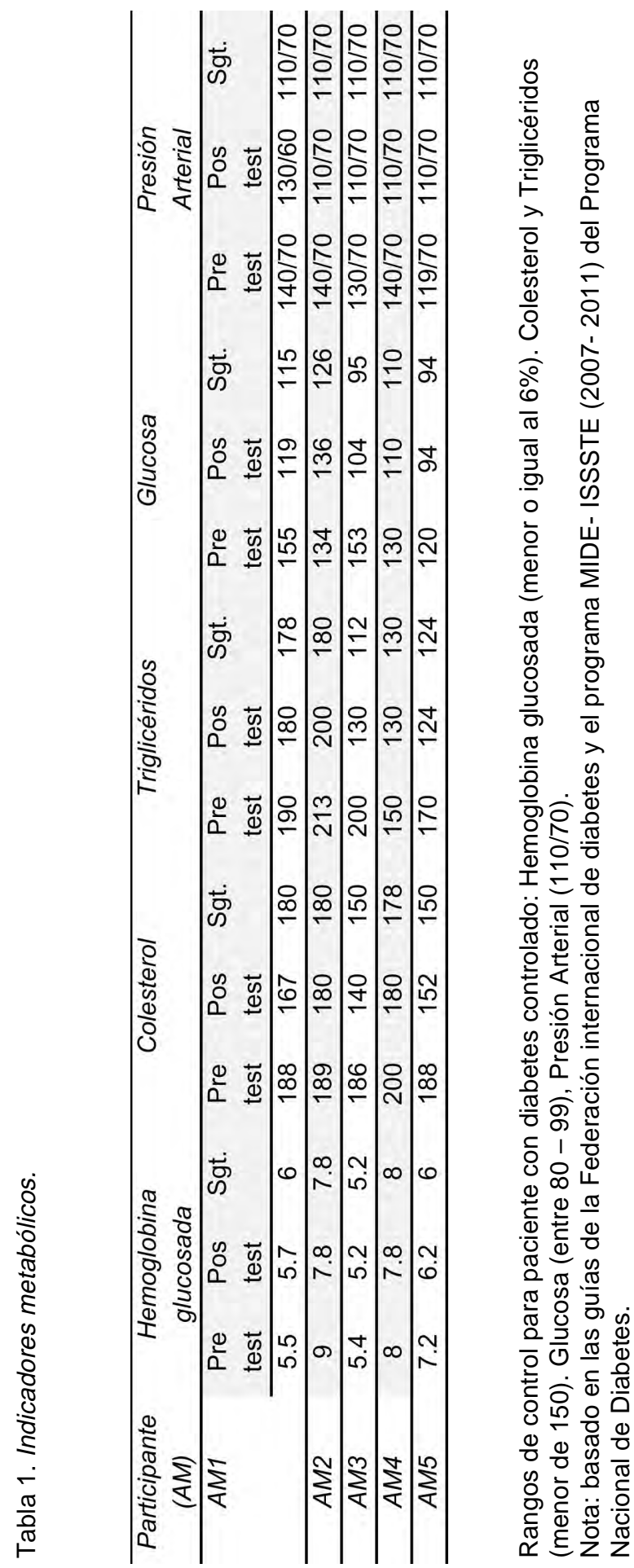


La BIBLIOFAM muestra tener efectividad como tratamiento psicoeducativo, mayormente en el control de grasas y presión arterial, aunque con resultados modestos; sin embargo, habrá que implementar un dispositivo para garantizar que los cambios continúen por más tiempo, para que impacte más eficientemente a todos los indicadores y no solo a algunos, quizá incorporando dentro de las sesiones ejercicios de medición de indicadores, diseño de menús en donde toda la familia participe, organización de una agenda para realizar actividades físicas también en conjunto con la familia, entre otros; de tal forma que pueda propiciar una verdadera educación participativa involucrando más al paciente y sus familiares como generadores de su propio aprendizaje al establecer un vínculo entre la teoría y la práctica, que si bien la BIBLIFOFAM basada en el aprendizaje significativo, perseguía este objetivo, no fue del todo concretado.

La evaluación de la calidad de vida incluyó seis áreas que valoran los siguientes aspectos:

Salud física: corresponde lo relacionado a energía y fatiga, dolor y malestar, sueño y descanso (González-Celis, 2009).

De acuerdo a los resultados (ver fig. 1), solo dos de los participantes mejoraron sus registros en esta área, concordando con lo que se describe en diversas investigaciones como la de Glasgow, Ruggiero, Eakin, Dryfoos y Chobanian (1997); Hervás, Zabaleta, De Miguel y Beldarrín, (2007); Mata, Roset, Badía, Antoñanzas y Ragel (2003); Valle Coronado, López, Martín, Real, Sánchez y Silveira (2011), sobre que los pacientes con diabetes tienen una tendencia a presentar resultados inferiores al ser evaluados en lo relacionado a salud física,

\section{Salud Física}

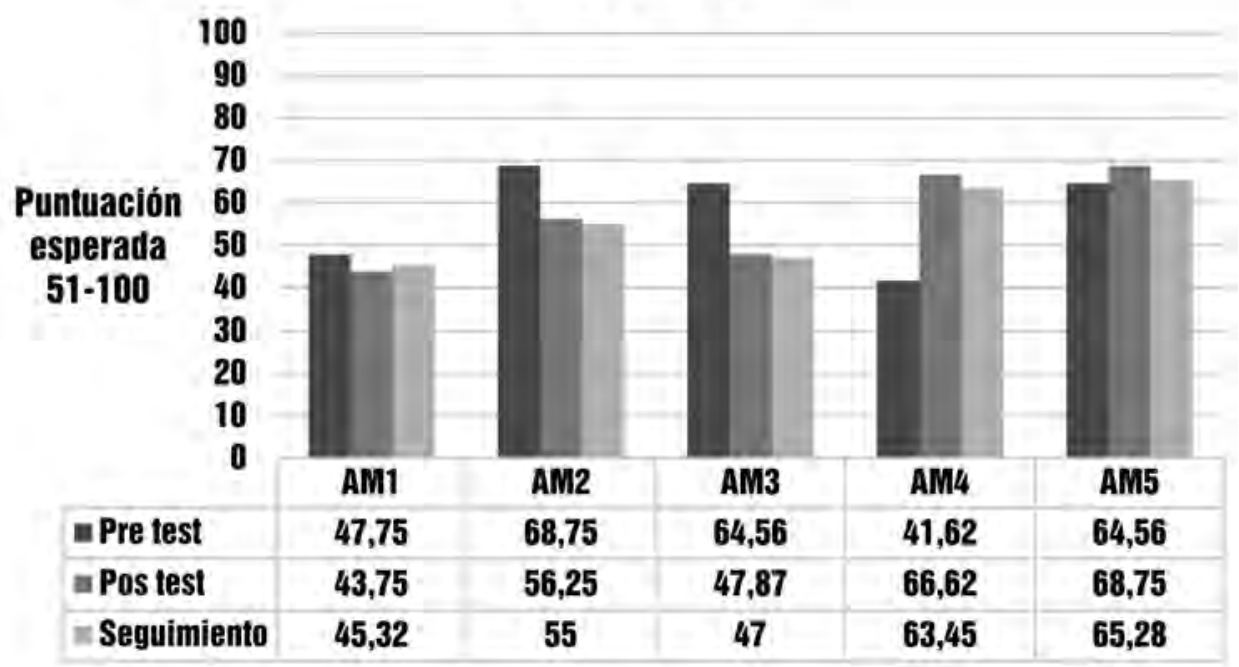

Fig. 1. Evaluación de la subescala salud física con relación a la calidad de vida del AM. 
malestar y dolor corporal. Por lo cual, se hace necesario incidir con intervenciones que impacten no solo en el control metabólico, lo cual es muy importante, pero que también busquen entender y modificar la vivencia de experimentar el proceso evolutivo de la diabetes, de tal forma que tenga un impacto en el desarrollo de comportamientos de autocontrol y autocuidado (Rhee, Cook, EL-Kebi, Lyles, Dunbar, Panayioto, Berkowitz, Boyd, Broussard y George, 2005).

La BIBLIOFAM, con respecto a la evaluación en lo relacionado a la salud física experimentada por los participantes, confirmó que este aspecto es el que se percibe como disminuido, no logrando con el tratamiento incidir en modificar la manera de experimentar la enfermedad, modificación que se viera reflejada a través de un mejor control y cuidado de sí mismos y por ende en un aumento en la autovaloración del estado de salud.

Salud psicológica: se evaluaron aspectos sobre imagen corporal y apariencia, sentimientos negativos y sentimientos positivos, autoestima, pensamientos, aprendizaje, memoria y concentración.

Con relación a ello, los resultados sobre la salud psicológica son distintos a la física (ver fig. 2), en este factor sí se lograron realizar mejoras, armonizando con lo ya descrito también por Alves, Silva, Lima, Brandão, Oliveira (2012), que describe cómo el conocimiento sobre la enfermedad tiene un impacto en su estado emocional, lo cual tiene una importante repercusión si lo que se persigue es que el paciente llegue a ser autónomo y eficiente en su autocuidado, y además se debe instar en abordajes psicoeducativos en los cuales se desarrollen este tipo de habilidades y destrezas.

\section{Salud Psicológica}

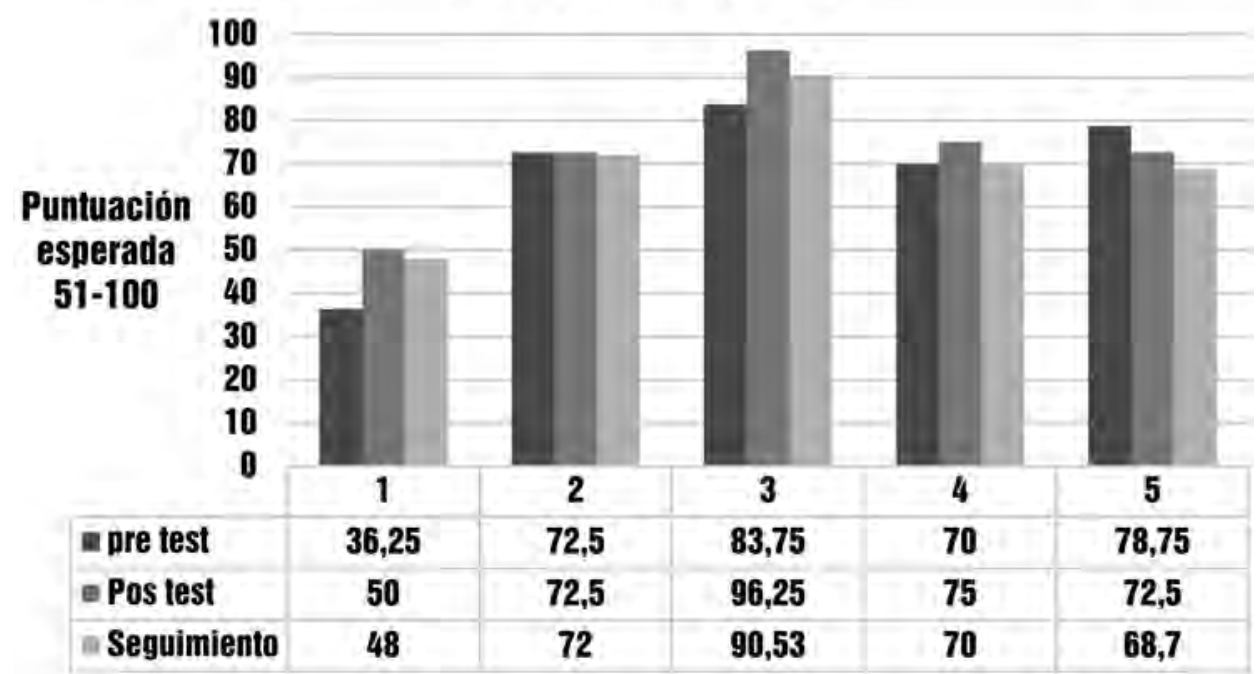

Fig. 2. Evaluación de la subescala salud psicológica con relación a la calidad de vida del AM. 
La BIBLIOFAM como intervención psicoeducativa desarrolló conocimientos específicos sobre el autocontrol y autocuidado de la enfermedad repercutiendo en un aumento, si bien moderado en la medición de la salud psicológica de los participantes.

Independencia: exploró lo relacionado con movilidad, actividades de la vida diaria, dependencia de sustancias médicas, asistencia médica y capacidad de trabajo.

Al respecto los resultados en esta dimensión muestran que en 4 de los 5 participante sí hubo un incremento (ver fig. 3), lo cual confirma que incidir en el mejor conocimiento de la enfermedad, dota de habilidades más eficientes para un mayor manejo del padecimiento y por consiguiente la sensación de autonomía (Acevedo, 2006, Fernández-Ballesteros, 2009; Tejada, Pastor y Gutiérrez, 2006;).

\section{Independencia}

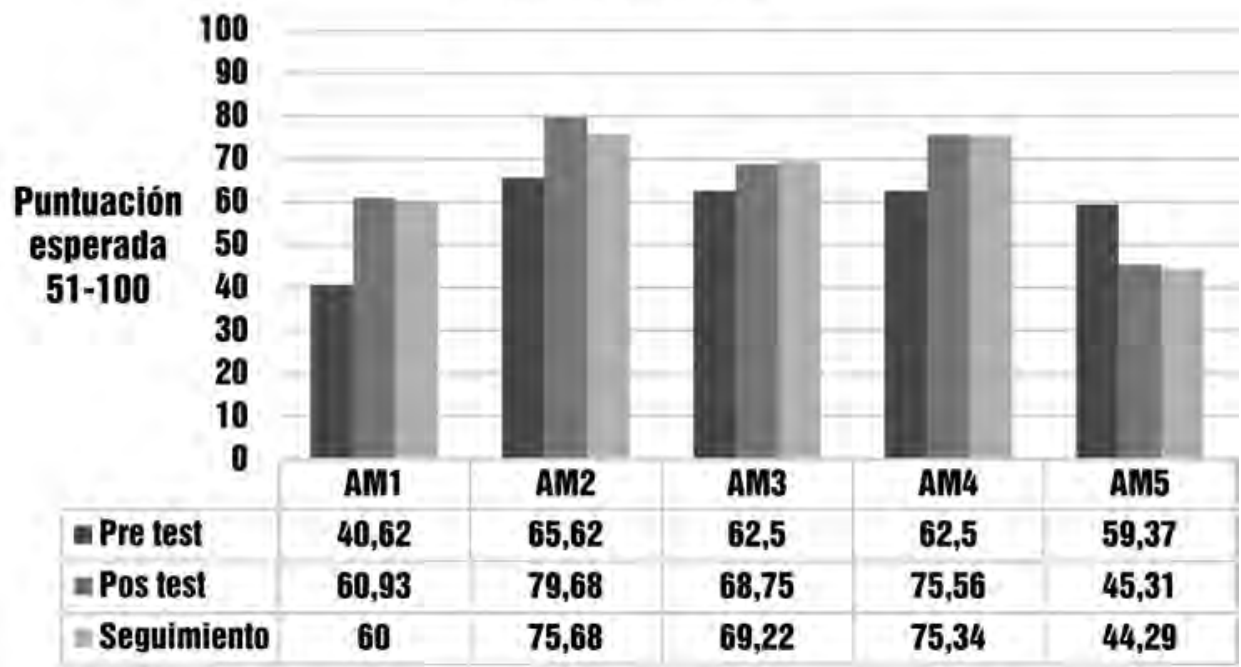

Fig. 3. Evaluación de la subescala independencia con relación a la calidad de vida del AM.

Aligual que Triado(2001) explica que apelar por la intervención psicoeducativa en las personas mayores y más aún si éstas padecen un proceso degenerativo crónico, se puede en los AM incrementan el bienestar y la confianza en sí mismos y de esta manera aminorar el estereotipo social de individuos apartados de la posibilidad de aprender y relegados a la mirada deficitaria de la vejez que con la perspectiva que promueve la psicogerontología.

La BIBLIOFAM como propuesta de intervención psicoeducativa tuvo en estos cinco casos específicos un impacto moderado en lo que refiere a mayor valoración de independencia en los AM participantes.

Relaciones sociales: en esta área se evaluaron las relaciones personales, soporte social y actividad sexual: con relación a ello, los resultados muestran un 
mínimo cambio en esta área, siendo los puntajes más bajos de la evaluación (ver. fig. 4). La posible explicación al respecto podría radicar en que su mayor apoyo reside en la familia, reduciendo su apoyo social secundario (vecinos, instituciones, comunidad). Esto concuerda con los hallazgos en otras investigaciones (Molina y Meléndez, 2007) en las que se muestra que las variables generadoras de bienestar están representadas por los factores familiares y sociales. Cava y Musitu (2000) encontraron que el mayor porcentaje de apoyo recibido por el AM proviene del ámbito familiar (cónyuge e hijo 63,8\%), y de parientes, amistades y vecinos $(8,1 \%$ ). Lo cual es algo positivo, pues afirman que la perspectiva familiar de la intervención no está equivocada, sin embargo, no puede responsabilizarse únicamente a la familia el bienestar social de su AM, sino que estos resultados invitan a revisar, analizar, y enriquecer la intervención de la BIBLIOFAM, a procesos psicoeducativos que generen la ampliación del entorno en que el AM pueda socializar, a través de fortalecer la red social de vecinos, co-etarios, instituciones y comunidad en general.

\section{Relaciones Sociales}

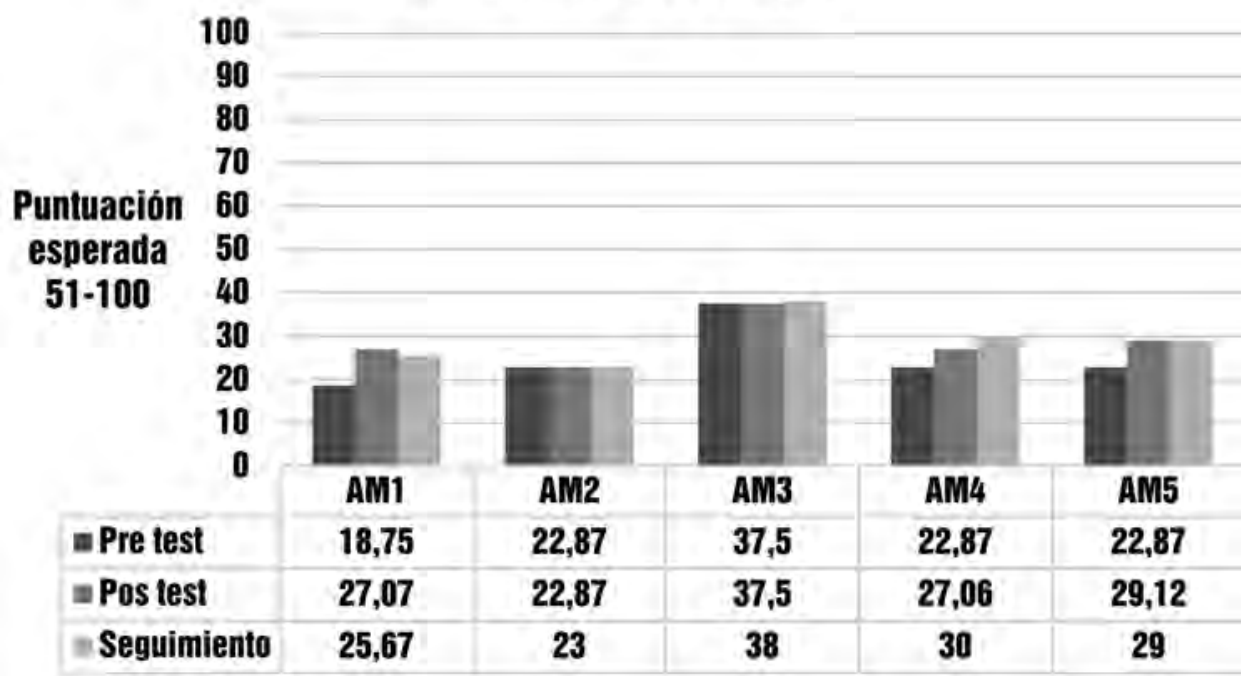

Fig. 4. Evaluación de la subescala relaciones sociales con relación a la calidad de vida del AM.

Las relaciones sociales de los AM participantes están circunscritas a su entorno familiar. La BIBLIOFAM pudo mejorar esta área de manera mínima, requiere mayor atención, si se desea trabajar desde el enfoque psicogerontológico, enfatizando la necesidad de apoyo al sistema familiar, por parte de la comunidad e instituciones con alternativas recreativas acordes a las necesidades del AM, para incidir en un envejecimiento saludable integral del AM con DM2.

Medio ambiente: se evaluó lo referente a recursos financieros, seguridad, ambiente físico (contaminación, clima, etcétera), transporte, recreación, tiempo 
libre, participación social.

Los resultados muestran que no hubo modificación en los registros previos, a los que se obtuvieron al término del tratamiento y posteriormente durante el seguimiento, sólo en uno de los participantes (ver fig. 5). Sin embargo, esto no difiere de lo encontrado en otras investigaciones (Vázquez-Honorato y SalazarMartínez, 2010; Landázuri Ortiz y Mercado, 2004; Mercado, 1998) en que se hace hincapié en las deficiencias que existe en la planificación de espacios inclusivos que contemplen las necesidades específicas de cada persona Instituto Nacional de la Personas Mayores-México (INAPAM, 2016).

\section{Medio ambiente}

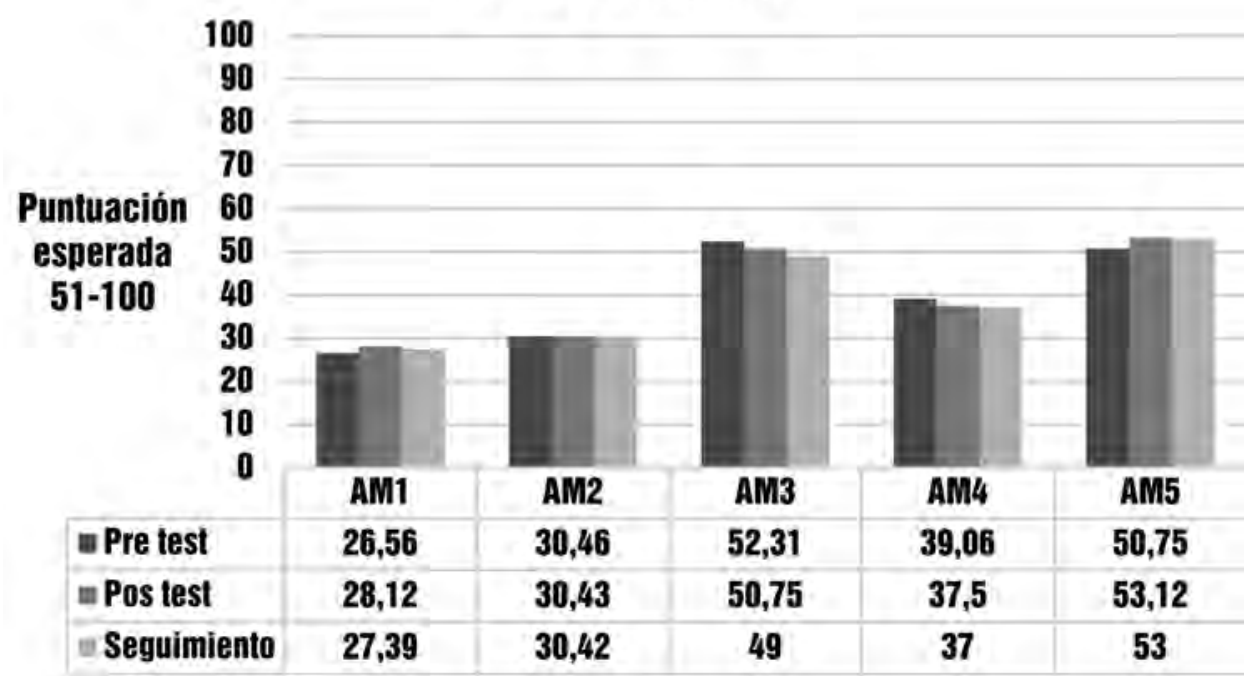

Fig. 5. Evaluación de la subescala medio ambiente con relación a la calidad de vida del AM.

De acuerdo con los resultados, queda manifiesto que el trabajo con los AM no solo puede ser trabajado de manera aislada, sino debe insistirse en la inclusión de otros sistemas además de la familia, en la responsabilidad del cuidado y atención del AM, de tal forma que pueda darse respuesta a sus necesidades de manera pronta y pertinente. Quizá pueda incluirse dentro de la temática de cuidado y autocuidado, la prevención de caídas, las modificaciones de arquitectura mínima que requiere una habitación para la prevención de accidentes, de tal manera que pueda tener una experiencia el AM de mayor confianza y confort.

Espiritual: en esta categoría se exploró lo relacionado con religión y creencias personales del AM. De acuerdo con lo reflejado en los resultados, se puede apreciar que se mantuvieron en tres de los participantes los mismos puntajes en el antes, después y seguimiento, y sólo en dos aumentó el puntaje (ver fig. 6). 


\section{Espiritual}

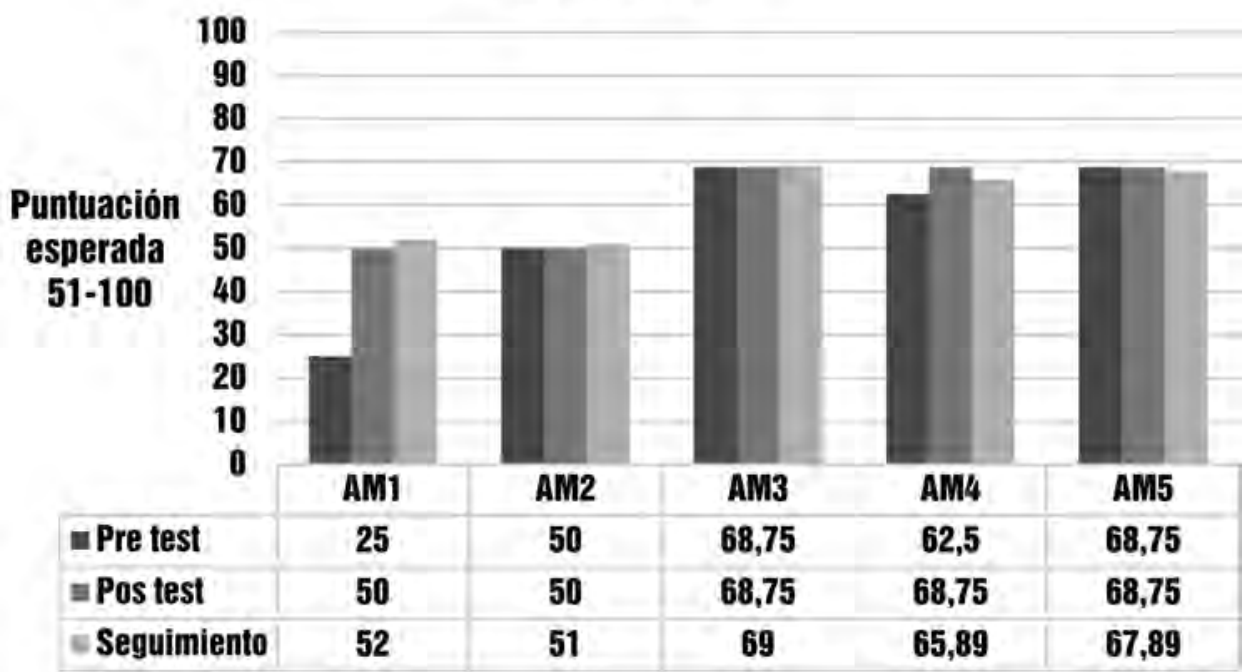

Fig. 6. Evaluación de la subescala espiritualidad con relación a la calidad de vida del AM.

No obstante, se evidencia que esta dimensión se calificó de manera positiva, lo cual refleja que influir en esta área puede tener repercusiones favorables en lo que se refiere al autocontrol y autocuidado de la enfermedad que padecen estos AM, dado que la espiritualidad contribuye a dar un sentido y propósito en la vida, $\mathrm{y}$ orientan la conducta de las personas, sus relaciones interpersonales y su forma de sentir y de pensar, tanto la realidad como a sí mismos. Es decir que este es un recurso por el cual se pueden invertir y potenciar estilos de vida y un envejecimiento saludable que repercute en una apreciación de mejor calidad de vida, tal y como lo refieren Kliever y Saultz (2006), Koenig (2001), Navas, Villegas, Hurtado y Zapata (2006), y Levin (2001). Y también en lo que refiere Delgado (2005), RiveraLedesma y Montero-López (2007; 2005) al haber encontrado beneficios de la espiritualidad en la salud, alivio del estrés de la hospitalización, salud mental, bienestar, enfrentamiento a la muerte, entre otros.

Con relación a la espiritualidad en el abordaje de los AM, es necesaria la pertinencia de incorporar esta dimensión en el asesoramiento o tratamiento de los adultos mayores, pues podría tener un impacto positivo en el abordaje de los procesos de salud-enfermedad. Esta dimensión no estaba contemplada en el tratamiento de la BIBLIOFAM, por lo que deberá analizarse la mejor manera de incorporarla en las sesiones que la conforman.

El concepto de autoeficacia, fue establecido por Bandura en 1977 y constituye el eje principal de la teoría social cognitiva (Bandura, 1986, 1999, 2001). De acuerdo a esta teoría, la motivación y la conducta del ser humano están reguladas por el pensamiento; en lo que se involucran tres tipos de expectativas: a) de la 
situación, en la que las consecuencias son producidas por eventos ambientales independientes de la acción personal, b) de resultado, que se refiere a la creencia que una conducta producirá determinados resultados y c) de autoeficacia, que se refiere a la creencia que tiene un individuo de poseer la capacidad para llevar a cabo acciones necesarias que le permitan obtener resultados deseados (Bandura, 1999).

Así mismo Bandura $(1999,2001)$ plantea que la autoeficacia percibida debe ser conceptualizada de manera específica, por ello en la variable autoeficacia se evaluó de manera específica actividades que debería realizar el AM, a continuación se analiza cada dimensión que la constituye:

Actividades individuales: Los resultados en esta dimensión muestran que sólo en uno de los participantes pudo elevar su puntuación al evaluarse posterior al tratamiento y seguimiento (ver fig. 7). En el resto esta apreciación disminuyó, por lo que no se logró uno de los objetivos primordiales de la BIBLIOFAM, dentro de lo que marca cualquier abordaje psicogerontológico, como ya lo mencionaban Losada Baltar y Montorio-Cerrato (2005); Montorio, Díaz-Veiga, e Izal (1995), sobre los programas para la atención de los AM, se enfatiza que ellos deben estar correctamente articulados en la red social y sanitaria, para promover un adecuado apoyo familiar, que genere autonomía e independencia en las personas mayores.

\section{Actividades individuales}

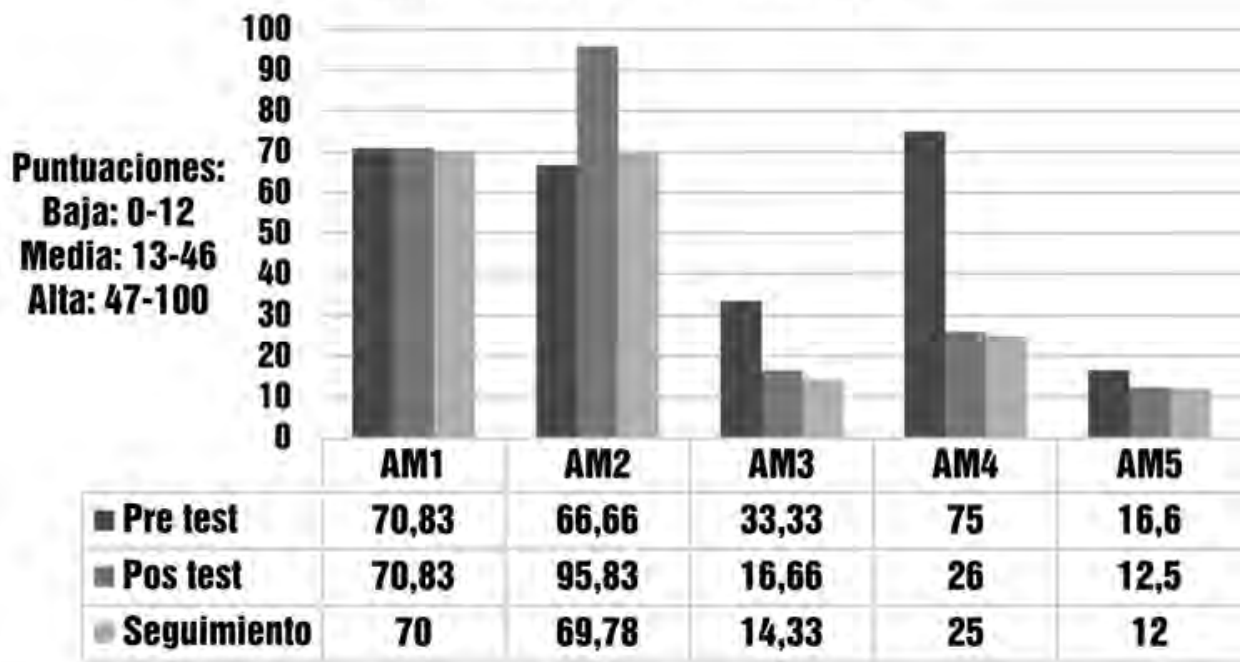

7. Fig. Evaluación de la subescala actividades individuales con relación al grado de autoeficacia del AM.

La importancia de lo anterior se basa en la buena adaptación de las personas mayores a su medio y su bienestar está fundamentalmente unido a la capacidad que tengan para realizar las actividades cotidianas esenciales. Por ello la OMS (1984) estableció que el número y calidad de años que una persona mayor puede vivir sin 
discapacidad se le denomina expectativa de vida autónoma, el cual es considerado como un indicador esencial de la salud, y por ende de calidad de vida de una población.

Sin embargo, es un hecho ineludible que un padecimiento crónico degenerativo como es la diabetes mellitus contribuyen de forma notable a la discapacidad y dependencia física (Montorio, 2007), pero en estos casos la dependencia puede ser prevenida con tratamientos adecuados, que contemplen la continua interacción entre variables biológicas y psicosociales, tales como los estereotipos, las atribuciones, las actitudes, las conductas de apoyo y de comunicación, la autoeficacia que pueden llegar a inducir dependencia y que muchas veces son compartidas por la sociedad, por ello las intervenciones deben contemplar al entorno social del AM como partícipe y en colaboración activa en el mantenimiento de la autonomía (DíazVeiga, Montorio y Yanguas, 1999).

La BIBLIOFAM, como programa psicoeducativo deberá enfatizar a profundidad los aspectos que puedan favorecer de manera adecuada el desarrollo de autonomía e independencia, en los AM que participen de esta intervención.

Actividades mentales: en la categoría en donde se evaluó la autoeficacia con relación a las actividades mentales que puede realizar el AM participante. Los resultados muestran que en tres de ellos los registros aumentaron sus puntajes al finalizar el tratamiento y durante el seguimiento (ver fig. 8). Esto tiene una connotación positiva pues coincide con los hallazgos de Feldberg y Stefani (2007), que afirma que la persona de edad con creencias positivas acerca de su capacidad para resolver tareas que implican la actividad mental se desempeñará muy proba-

\section{Actividades mentales}

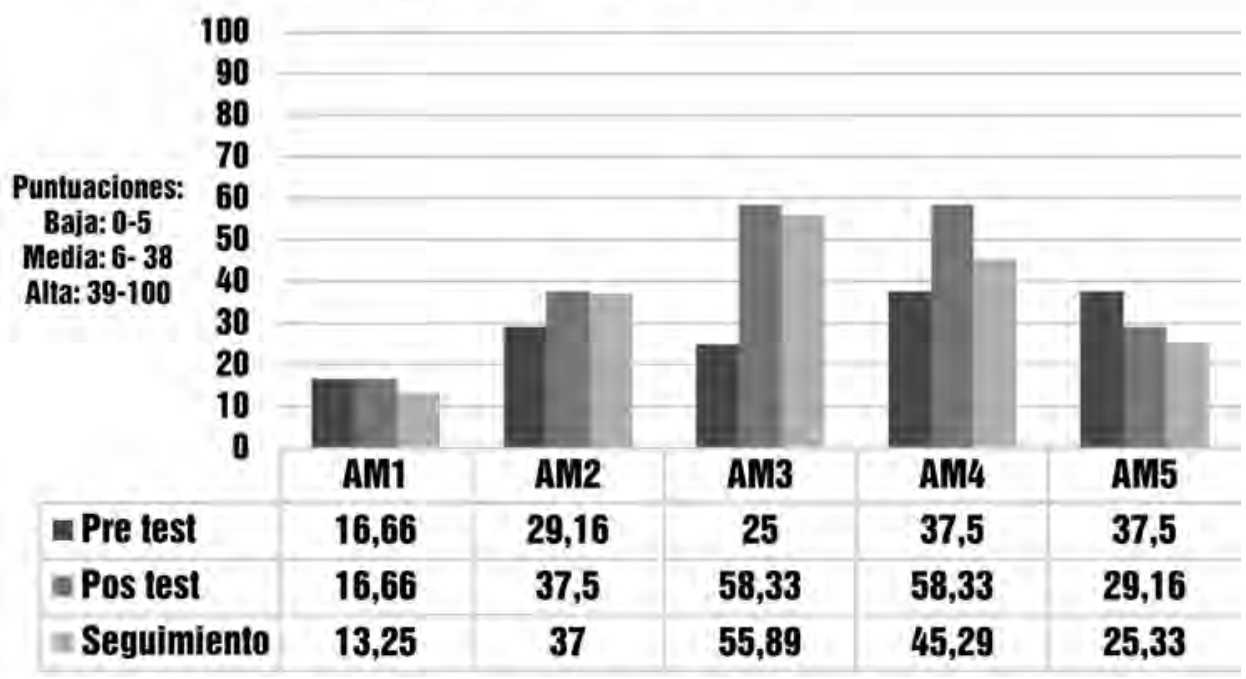

Fig. 8. Evaluación de la subescala de actividades mentales con relación al grado de autoeficacia del AM. 
blemente en forma exitosa; mientras que aquéllos con creencias de autoeficacia negativas en esta actividad, obtendrán un rendimiento bajo en dicha tarea. El sistema de creencias del AM resulta ser central pues incluso determina su participación social en tareas de tipo recreativo y de protección a la salud (Everard, Lach, Fisher y Baum, 2000).

Las personas mayores con autoeficacia positiva en las tareas mentales optan por realizar con mayor frecuencia la lectura de libros, diarios y revistas, escuchan la radio, miran televisión, les agrada asistir al cine y teatro, participan en juegos de mesa, asisten a exposiciones de arte, o coleccionan objetos, que aquellos que tienen creencias de baja autoeficacia en esta área (Menec, 2003). Lo cual retroalimenta el diseño de la BIBLIOFAM, pues estuvo basado en lecturas y actividades de aprendizaje que implicaron mayor trabajo en este factor.

En especial la BILIOFAM, por su diseño propició el trabajo de autoeficacia en actividades mentales de los AM participantes, lo cual es alentador pues es un recurso potencial para la generación de un envejecimiento exitoso y actividades protectoras de salud. Sin embargo, el impacto fue mínimo, por lo que habrá que revisar nuevamente el contenido de las lecturas e incrementar actividades que incidan de manera más eficaz en esta área, como por ejemplo incorporar en las sesiones actividades lúdicas recreativas: ajedrez, domino, baraja, entre otros.

Actividades físicas: con relación a los resultados que se obtuvieron en esta categoría, puede apreciarse que esta actividad estaba presente sólo en un participante, en tres fueron puntuaciones muy bajas (ver fig. 9).

\section{Actividades físicas}

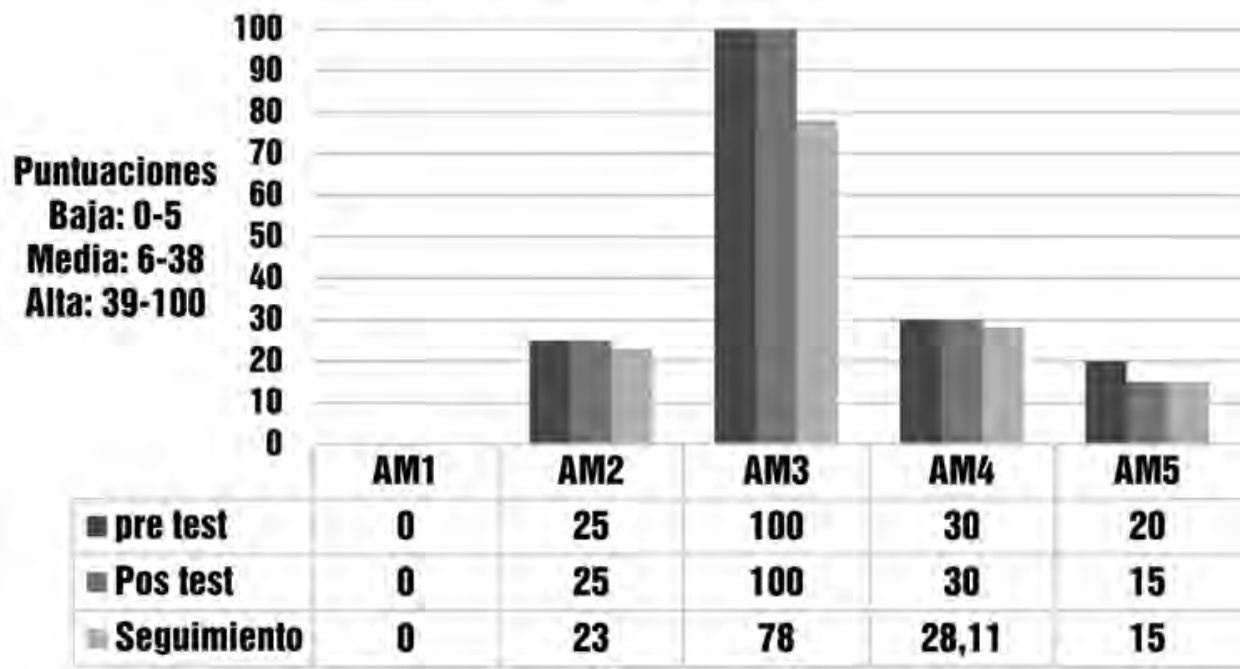

Fig. 9. Evaluación de la subescala actividades físicas en relación con el grado de autoeficacia del AM. 
Al respecto Bandura (1986) ya señalaba que el desarrollo de la autoeficacia, con base en la actividad física, tenía una fase crítica en la tercera edad debido a la pérdida de capacidades personales y funciones sociales. También en la investigación de Reigal y Videra (2013), sugiere que de la estabilidad en el tiempo que mostrarían aquellas personas que son físicamente activas, dependería el desarrollo de esta área, lo cual concuerda con la puntuación del único participante, el cual ha sido deportista toda su vida.

Por lo cual la autoeficacia puede ser una herramienta para llevar a cabo otras conductas de salud, por lo que promoverla, estará ayudando directamente a conseguir los propósitos de autocuidado y mejor autocontrol de la diabetes (Bandura, 1999; Garrido, 2000). Además, como lo sugieren Menéndez y Brochier (2011) es una necesidad posibilitar estrategias de intervención que contribuyan al envejecimiento activo con el fin de prevenir las situaciones de dependencia que pueden ser ocasionadas por factores físicos o biológicos pero que, en general, tienen que ver con cuestiones psicoafectivas que afectan de manera directa a las capacidades fisicas de los AM.

La BIBLIOFAM, en su diseño contempla información con respecto el autocuidado que deben realizar los pacientes de DM2, con especial énfasis en las actividades físicas; sin embargo, no alcanzó su desarrollo como se esperaba, por lo que se deberá reforzar este aspecto con algún dispositivo que garantice de mejor manera su ejecución. Como anteriormente ya se describía, desarrollando de manera familiar y en conjunto una agenda en la cual haya convivencia como grupo y sean actividades recreativas, pero a la vez implique la activación física: caminatas juntos, paseos en bicicleta, natación, entre otras.

Actividades sociales: en los resultados de esta categoría, sólo uno de los participantes logró alcanzar los puntajes óptimos (ver fig. 10). Algo que puede explicar este resultado es el hecho, como se había mencionado anteriormente, en la categoría denominada relaciones sociales, que los AM participantes circunscriben sus relaciones y actividad social al ámbito familiar, lo cual pone de manifiesto que para esta población etaria, existe mayor dificultad para lograr satisfacción de esta área, ya que influye, de alguna manera, el deterioro físico que les impide entrar en contacto con otras personas; lo que genera una reducción en forma considerable de su red de relaciones interpersonal y una ruptura con la redes sociales, lo que afecta su inclusión social y su bienestar emocional, generándose una situación de soledad emocional y social (López, 2010; López, 2005).

Aunado a lo anterior, es importante de fortalecer la autoeficacia en esta área, ya que las personas mayores eligen, inician y desarrollan las actividades que se perciben capaces de realizar exitosamente, por lo cual se hace imperiosa ampliar esta autovaloración (Salvarezza, 1998).

Por lo anterior, la intervención expone la necesidad de incurrir no sólo en el ámbito familiar sino ampliar esta red de apoyo, pero sobre todo ratifica la necesidad de incluir planes y políticas adecuadas que garanticen la inclusión de los AM en 


\section{Actividades sociales}

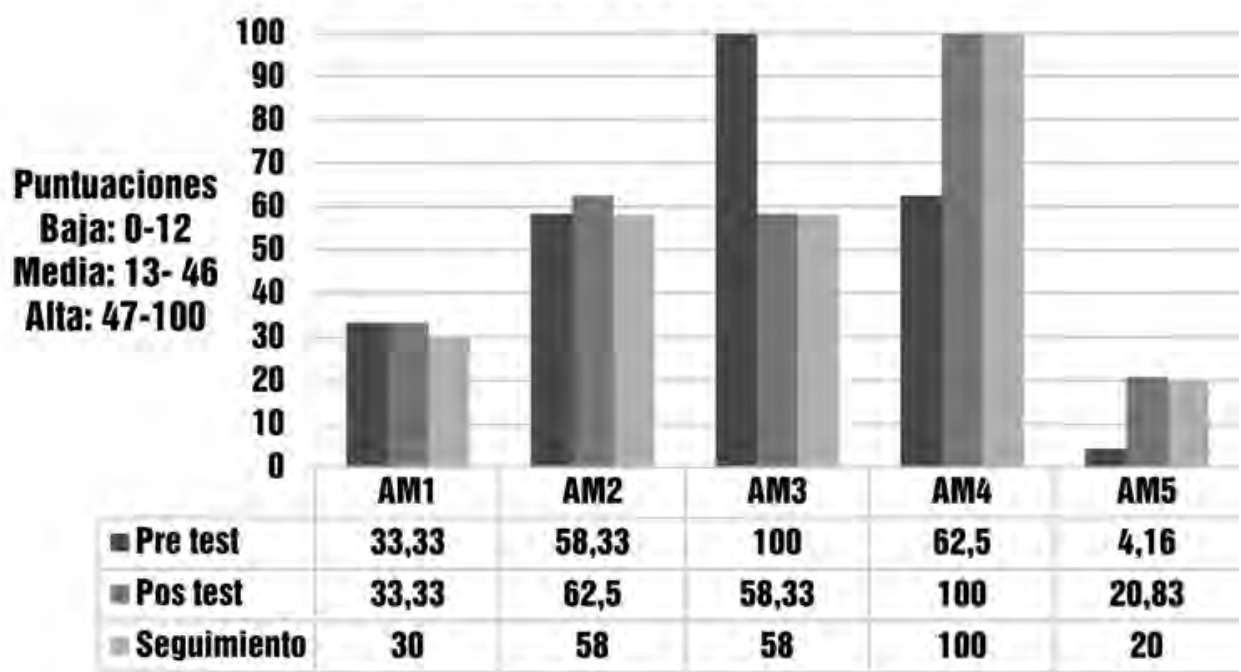

Fig. 10. Evaluación de la subescala actividades sociales con relación al grado de autoeficacia del AM.

forma activa a las actividades de índole social, que garanticen la satisfacción de sus necesidades de interacción personal amplia.

Actividades de protección a la salud: de acuerdo a los resultados que se reportaron en las evaluaciones, antes, después y en el seguimiento, en esta categoría se observa que los registros son altos en sólo dos de los participantes y como resultado de la intervención, los otros tres participantes tenían esta área ya desarrollada previa al tratamiento (ver fig. 11), aun así los resultados concuerda con lo que se describe en la investigación de Azzollini, Bail Pupko y Vidal (2012), Persini (2011) y Rubin (2000) en las que se explica que a mayor información existe una mayor tendencia a mejorar las practicas saludables. Sin embargo, Amigó, Fernández y Pérez (1998), así como Díaz, Galán y Fernández (1993) también señalan que la mayoría de los pacientes con diabetes, a pesar de conocer las pautas de tratamiento para un buen autocuidado, tienen dificultades para seguirlo ininterrumpidamente en relación a prácticas de ejercicio físico, autoanálisis de glucosa y cumplimiento de dieta (Rubio Llorente y López Vergara, 2003), lo cual evidencia que no se trata sólo de atender el componente psicoeducativo, sino también dar importancia al aspecto emocional para afrontar la cronicidad y la aceptación de un nuevo estilo de vida, de un modo totalmente diferente del que se realizaba con anterioridad al diagnóstico (Gordon y Benishek, 1996).

La BIBLIOFAM, a través de la información dada proporcionó a los participantes conocimientos específicos para el cuidado y mayor control de la enfermedad. Sin embargo, habrá que llevar a la práctica real y pasar del plano cognitivo al de la ejecución de la acción concreta objetiva que evidencie la modificación de compor- 


\section{Actividades de protección a la salud}

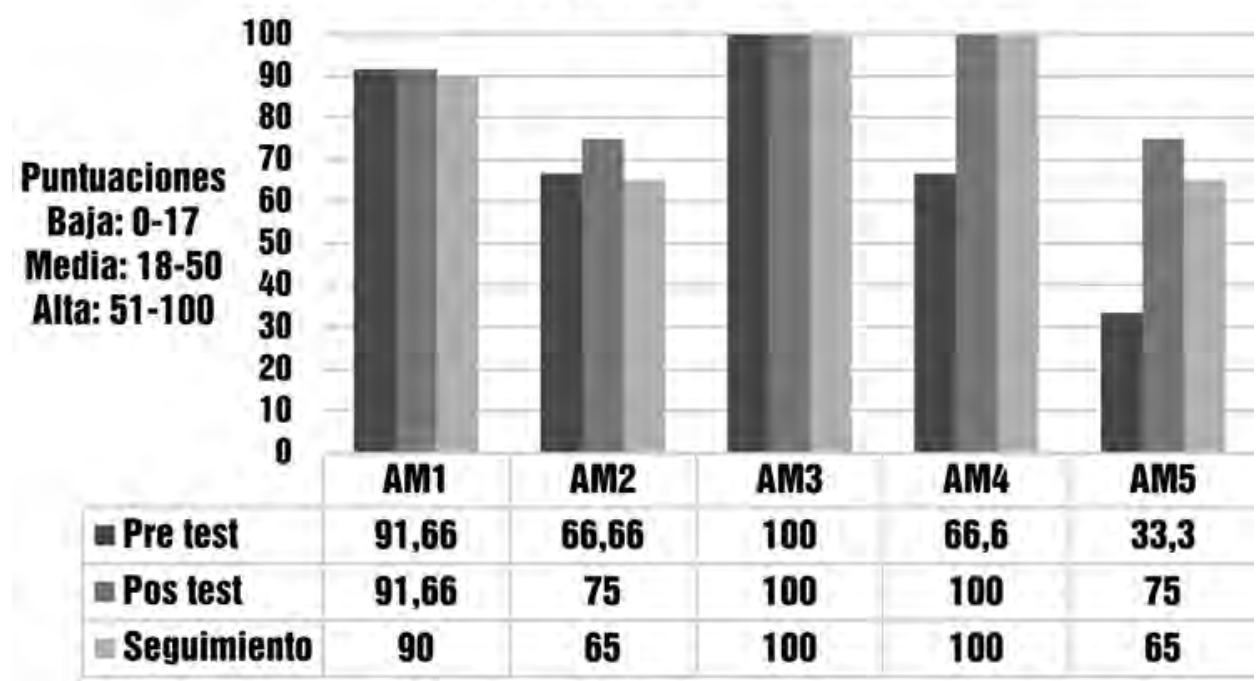

Fig. 11. Evaluación de la subescala de actividades de protección a la salud en relación con el grado de autoeficacia del AM.

tamientos a estilos saludables.

Los resultados registrados en la variable sobrecarga del cuidado, muestran que hubo disminución mínima, en tres de los participantes, solo en uno su cambio fue notable en 20 puntos que registraba sobrecarga intensa a pasar a la no existencia de sobrecarga y solo en uno de ellos permaneció la sobrecarga (ver fig. 12). Según

\section{Sobrecarga del cuidador}

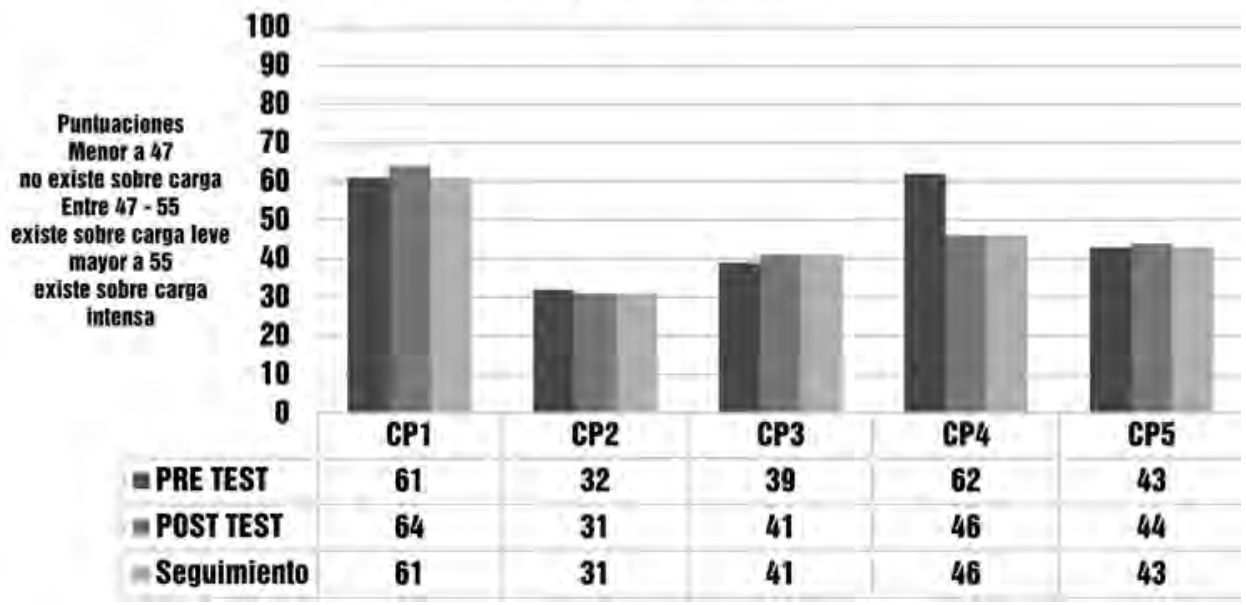

Fig. 12. Evaluación de la sobrecarga del CP. 
Losada-Baltar y Montorio-Cerrato (2005) las intervenciones de corte psicoeducativo con cuidadores informales de AM han mostrado eficacia en los siguientes puntos:

- Los cuidadores aprenden habilidades y estrategias que ayudan a aminorar el proceso de deterioro por la enfermedad.

- Con las intervenciones los cuidadores reciben beneficios psicológicos, que se reflejan en una mejor salud fisica y respuesta inmmunologica.

- El beneficio mayor por la participación de intervenciones psicoeducativas son las personas objeto del cuidado.

- En el caso de procesos de demencia, se retarda el ingreso del familiar a una institución.

Por otro lado Zabalegui, Navarro, Cabrera, Fernández-Puebla, Bardallo, Rodríguez, Gual, Fernández y Argemí (2008) refieren que la eficacia de las intervenciones dirigidas a los CP sobre las variables ansiedad y depresión demuestran ser más eficaces para disminuir esta sintomatología a corto plazo, aunque el efecto positivo disminuía con el transcurso del tiempo, coincidiendo con lo que ya describían Akkerman y Ostwald (2004).

Sin embargo, en el estudio que se llevó a cabo para valorar las necesidades percibidas por los CP de ancianos dependientes, Zabalegui et.al. (2008) encontraron que las demandabas apuntaban a la obtención de más recursos sociales para afrontar el cuidado de un anciano dependiente, la dificultad en el acceso a los escasos recursos disponibles y la escasa información que se recibe sobre el proceso de enfermedad de su familiar. El aspecto que los cuidadores valoraron de forma positiva fue el apoyo que recibían de los profesionales sanitarios, de quienes resaltaban su dedicación, capacidad de compromiso y escucha.

Finalmente Pinquart y Sörensen (2006) recomiendan en el diseño y desarrollo de las intervenciones dirigidas al CP, proveer conocimientos y ayudar en la adquisición de habilidades para facilitar la adaptación de las personas al proceso de su enfermedad o la de sus familiares. Actualmente, los programas terapéuticos abordan al paciente y al CP como la unidad a tratar, sin dejar de lado el contexto tanto físico y social. El objetivo de estas intervenciones es disminuir la sintomatología negativa (sobrecarga, ansiedad y depresión), facilitando así la permanencia del anciano dependiente en su entorno familiar.

De acuerdo a lo anterior la BIBLIOFAM, con base en su diseño, da respuesta a las necesidades que han sido documentadas en las investigaciones citadas, proporciona información sobre el proceso de la enfermedad de su familiar, toma en cuenta su contexto físico y social, además de que por su implementación facilita la comunicación entre los participantes, con la ayuda de la orientación terapéutica, generando feedback de los estados de ánimo del CP y el familiar enfermo, y los demás integrantes de la familia. Sin embargo, los resultados han sido muy modestos por lo que hace falta enfatizar aspectos que realmente ayuden a los cuidadores primarios a disminuir la carga del cuidado. Lo que da la oportunidad de revisar, analizar cada actividad de las sesiones encaminadas a este objetivo, para corregirlo 
o enriquecerlo.

Con relación a la configuración familiar, los resultados mostraron menor impacto en las áreas que se definen como problemáticas, tales como hostilidad y evitación de conflictos, y mando y problema de expresión de sentimientos (ver fig. 13-16), concordando con el perfil de las familias con comportamientos psicosomáticos los conflictos que viven en el interior del sistema, como lo describe Minuchin (1982); Minuchin y Fishman (2001); Minuchin, Nichols y Lee (2011); Minuchin, Rosman y Baker (1978) y Selvini, Cirillo, Selvini y Sorrentino (1990). Aun así, en las áreas de ambiente familiar y cohesión familiar, que son los aspectos positivos de las familias, se pueden potenciar recursos que ayuden a modificar las formas de interacción evitando así la utilización del síntoma para entablar vínculos afectivos.

\section{Ambiente familiar}

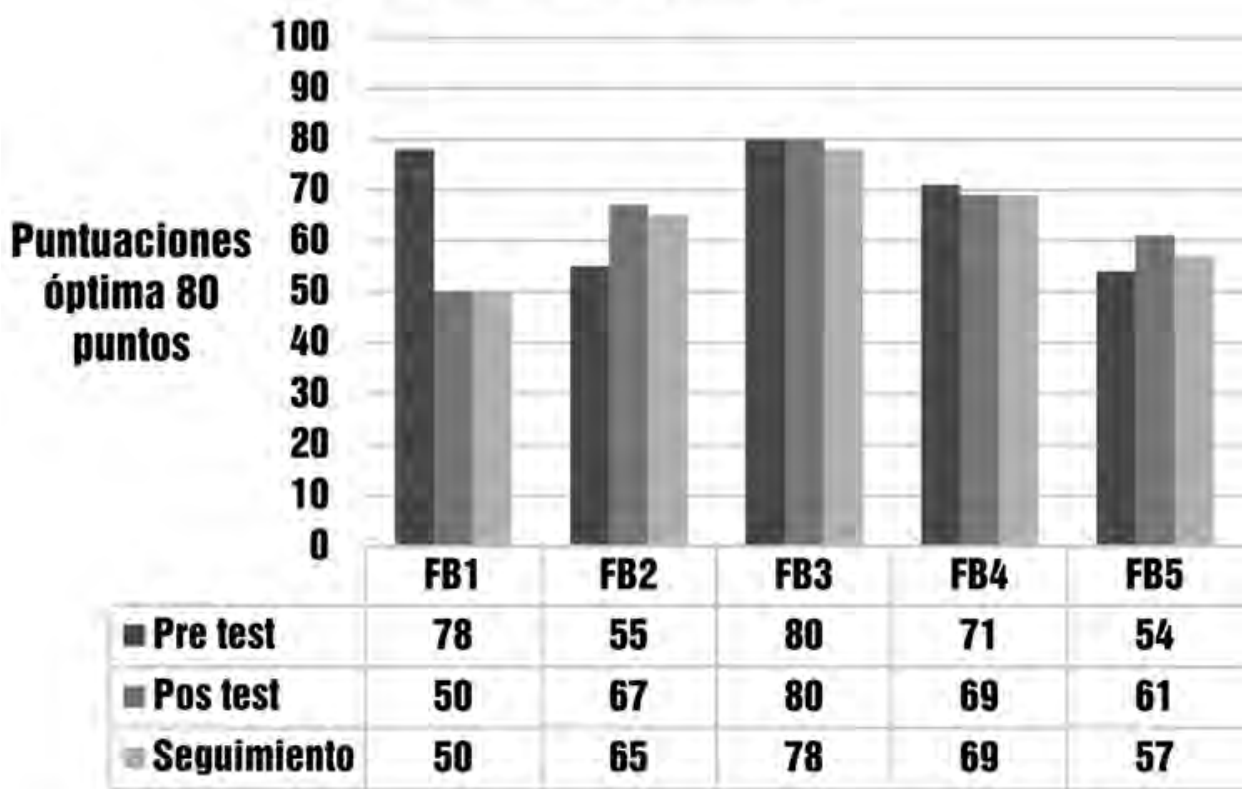

Fig. 13. Evaluación de la subescala ambiente en relación al funcionamiento familiar. 


\section{Hostilidad / evitación de conflicto}

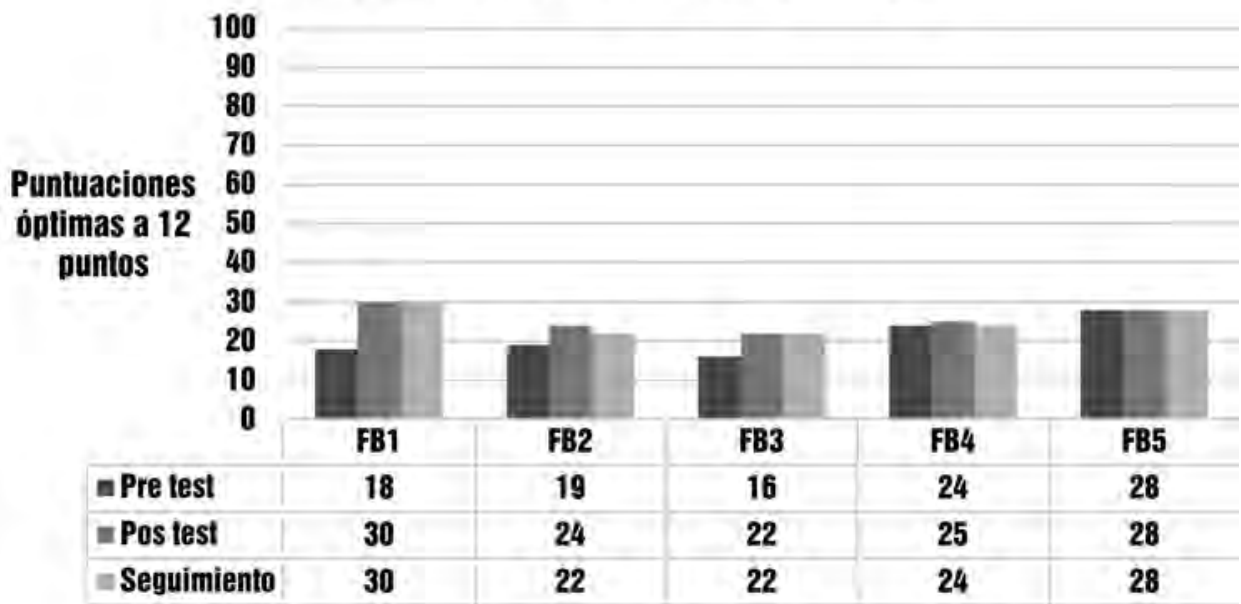

Fig. 14. Evaluación de la subescala hostilidad/evitación de conflicto con relación al funcionamiento familiar.

\section{Mando / problema de expresión de sentimientos}

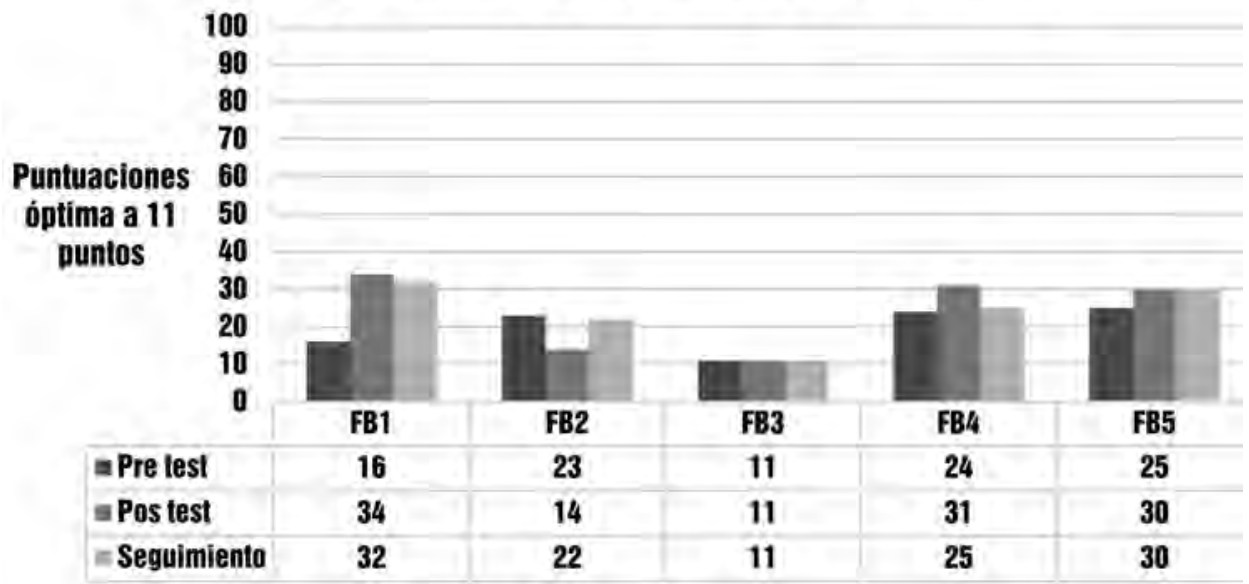

Fig. 15. Evaluación de la subescala mando/problema de expresión de sentimientos con relación al funcionamiento familiar. 


\section{Cohesión familiar}

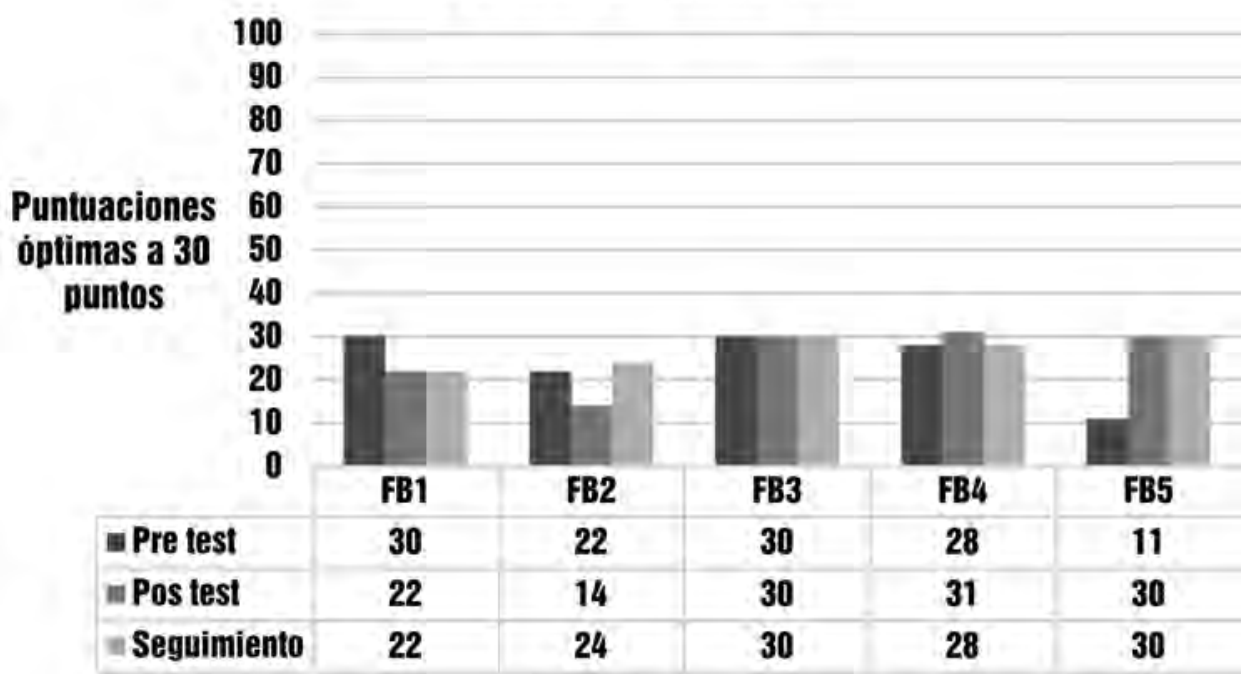

Fig. 16. Evaluación de la subescala cohesión con relación al funcionamiento familiar.

López, López, Ruiz, López y Escobar (2004), refieren que en el control del padecimiento de la diabetes, intervienen variables psicosociales que influyen en las fluctuaciones de la glucemia. La enfermedad es percibida como un factor que vulnera la estabilidad de la familia y a menos que el ambiente familiar cambie para apoyar al paciente, gradualmente declina su nivel de autocontrol y cuidado (Robles, Mercado, Ramos, Alcántara y Moreno, 1995).

En ese mismo estudio realizado por López et. al. (2004), refieren que los pacientes con disfunción familiar mostraron un grado de conocimiento muy bajo, lo que puede estar relacionado con falta de apoyo del grupo familiar hacia el enfermo.

Con respecto a lo anterior, las intervenciones deben enfocarse a brindar la mayor y mas amplia información a la familia para que puedan estar preparados para cualquier contingencia, pero sobre todo para poder ayudar al familiar con diabetes a su buen cuidado y control (Fuentes y Maya, 2001; García y Suárez, 2006; Rubio y López, 2003;).

Por lo anterior se puede concluir que la BILIOFAM, en su diseño sí brinda las herramientas para que la familia en conjunto pueda estar informada sobre cómo apoyar y ayudar a su familiar con diabetes en el cuidado y autocontrol de la enfermedad, sin embargo, habría que implementar estrategias más precisas y no sólo informativas, que incidan en la modificación de la interacción interna de la familia para lograr una configuración más adecuada. 


\section{Conclusiones de la BIBLIOFAM}

El método basado en la psicoeducación, de la BILIOFAM, de acuerdo a la teoría de Ausubel (2002), se considera adecuado para habilitar con información y desarrollo de conocimientos al paciente y su familia para enfrentar lo que conlleva el cuidado y autocontrol de la diabetes, ya que se enfatizar en el aprendizaje significativo que logran los individuos, a través de las etapas que conforman el proceso cognitivo fundamentado en todos y cada uno de los elementos, factores, y condiciones que garantizan la adquisición, la asimilación, retención y aplicación del contenido de las lecturas, seleccionadas para la BIBLIOFAM, y que se ofrecieron a las familias participantes, de modo que adquirieran significado para ellos mismos, basado en el diseño de cada sesión que conformo la BIBLIOFAM. Como se describió anteriormente, yendo de actividades sencillas a más complejas, sin embargo, faltó tener mayor control y claridad en los mecanismos que garantizaran la consecución de este proceso cognitivo que se menciona, por lo que constituye una limitación y a la vez una sugerencia para su autoevaluación y reestructuración a partir del rediseño y mejora.

Al ser un tratamiento de corte educativo, su impacto debe observarse otorgándole un rango de tiempo más extenso para la verificación de su eficacia; puesto que la teoría reafirma que este tipo de intervenciones son las más adecuadas para el tratamiento de la diabetes y la única eficaz para el control de la enfermedad y la prevención de sus complicaciones (OMS, 1991), y más aún cuando la familia y no solo el individuo forma parte del abordaje (González Pinto, Arrillaga, Barbeito Rosa y Vega Pérez, 2010).

Además la BIBLIOFAM como tratamiento de psicoeducación familiar, tuvo como objetivo la intervención desde un punto de vista teórico y práctico, de tal forma las familias que la recibieron comprendieran la diabetes, su curso y pronóstico, enfatizando en aquellas variables que afectan a la misma, y agregando a cada una de ellas estrategias para hacer frente desde una perspectiva de la psicología educativa en salud; con un enfoque que ahonda en los factores sociales y psicológicos que afectan el desarrollo del padecimiento, muchos de los cuales se generan en la propia familia y repercuten de una $\mathrm{u}$ otra forma en la salud del individuo (Méndez-López, Gómez-López y Navarrete-Escobar, 2004), ya que comparten la experiencia de padecer la enfermedad, aunque desde perspectivas diferentes; pero ambos en interrelación.

\section{Limitaciones}

Faltó la implementación de dispositivos claros, que pudieran garantizar la modificación de manera eficaz en todos y cada una de las variables y no solo en algunos y con resultados mínimos. A su vez que estos mecanismos pudieran garantizar el cambio continuo incluso posterior a la etapa del seguimiento.

La sola verificación de la comprensión y entendimiento de las lecturas que se realizaban en las sesiones, a través de la bitácora de lectura, no fue lo suficiente para 
garantizar cambios importantes en las mediciones de las variables, por lo que se requiere modificar su uso como herramienta evaluadora o implementar otra de mayor eficacia.

\section{Recomendaciones}

Realizar una valoración en todo el diseño para mejorarlo y volver a someter a jueceo, piloteo e implementación. Atendiendo con análisis claro los indicadores que no resultaron beneficiados por la BILIOFAM, e incluso sobre los que mostraron cambios para lograr que éstos puedan ser significativos. Buscar la evaluación de las lecturas sugeridas y valorar si son las más adecuadas o incluir otras que sean más efectivas. Así también incluir actividades que conlleve la articulación de la teoría y la práctica, e incluir rejillas de autoevaluación.

\section{Referencias bibliográficas}

Acevedo, R. (2006). Calidad de vida. Argentina: Lugar.

Akkerman, R. y Ostwald, S. (2004). Reducing anxiety in Alzheimers disease family caregivers: the effectiveness of a nine-week cognitive-behavioral intervention. American Journal of Alzheimer's Disease \& Other Dementias, 19, 117-23.

Alves, P. D., Silva, C. C. N. M., Lima, S. A. L., Brandão, V. P. C. J. y Oliveira, Z. C. R. (2012). Efectos de intervención educativa sobre el conocimiento de la enfermedad en pacientes con diabetes mellitus. Revista Latinoamericana Enfermagem, 20(3), 1-8.

Amigó, I., Fernández, C. y Pérez, M. (1998). La adhesión a los tratamientos terapéuticos. En I. Amigó (ed.) Manual de Psicología de la Salud. Madrid (pp. 229-70), España: Pirámide,

Ausubel, D. P. (1982, 2002). Adquisición y retención del conocimiento. Una perspectiva cognitiva. Barcelona, España: Paidós.

Azzollini, S. C., Bail Pupko, V.y Vidal, V. A. (2012). El apoyo social y el autocuidado en diabetes tipo 2. Anuarios de Investigación, 19(1), 109-113.

Bandura, A. (1986). Social foundations of thought and action: A Social Cognitive Theory. Englewood Cliffs, New Jersey, NJ: Prentice Hall.

Bandura, A. (1999). Autoeficacia: cómo enfrentamos los cambios de la sociedad actual. Bilbao, España: Desclée de Brouwer.

Bandura, A. (1999). Ejercicio de la eficacia personal y colectiva en sociedades cambiantes. En Autoeficacia: cómo enfrentamos los cambios de la sociedad actual. España: Desclée de Brouwer.

Bandura, A. (2001). Guía para la construcción de escalas de autoeficacia. Revista Evaluar, 2(15), 7-37.

Calderón, T. J., Solís, V. J., Castillo, S. O., Cornejo, A.P., Figueroa, V., Paredes, J., Manrique, H. y Neyra, L. (2003). Efecto de la educación en el control metabólico de pacientes con diabetes tipo 2 del Hospital Nacional Arzobispo Loayza. Revista de la Sociedad Peruana de Medicina Interna,16(1), 17-25.

Cava M. J. y Musitu, G. (2000). Bienestar psicosocial en ancianos institucionalizados y no institucionalizados. Revista Multidisciplinar de Gerontología, 10, 215-221.

Delgado, C. (2005). A Discussion of the Concept of Spirituality. Nursing Science Quarterly, 18(2), 157-162.

Díaz-Veiga, P., Montorio, I. y Yanguas, J. (1999). Intervenciones con cuidadores de personas mayores. En M. Izal e I. Montorio (eds.), Gerontología Conductual. Bases para la intervención y ámbitos de aplicación. Madrid, España: Síntesis.

Díaz, L., Galán, S. y Fernández, G. (1993). Grupo de autocuidado de diabetes mellitus tipo 2. Salud Pública de México, 35(2), 169-176.

ENASEM, Estudio Nacional de Salud y Envejecimiento en México, (2012). Archivos de Datos y Documentación (uso público). Estudio Nacional de Salud y Envejecimiento en México. Recuperado de: www.ENASEM.org en [9 de febrero, 2015]. 
Everard, K. M., Lach, H. W., Fisher, E. B. y Baum, M. C. (2000). Relationship of activity and social support to the functional health of older adults. The Journals of Gerontology Series B: Psychological Sciences and Social Sciences, 55(4), pp. 208-212.

Feldberg, C. y Stefani, D. (2007). Autoeficacia y rendimiento en memoria episódica verbal, y su influencia en la participación social de las personas de edad. Anales de Psicología, 23(2), 282-288.

Fernández-Ballesteros, R. (2009). Envejecimiento activo. Contribuciones de la psicología. Madrid, España: Pirámide.

Fernández, V. A., Abdala, C. T. A., Alvara, S. E. P., Tenorio, F. G. L., López, V. E., Cruz C., S., Dávila, M. R., y González, P. A. A. (2012). Estrategias de autocuidado en pacientes con diabetes mellitus tipo 2. Revista de Especialidades Médico-Quirúrgicas, 17(2), 94-99.

Fuentes, G. M. C. y Maya, M. U. (2001). Atencioìn a la familia: la atencioìn familiar en situaciones concretas. Anales del Sistema Sanitario de Navarra, 24(2), 83-92.

García, M. M., Rivera, S., Reyes-Lagunes. I. y Díaz-Loving. R. (2006). Construcción de una escala de funcionamiento familiar. Revista Iberoamericana de Diagnóstico y Evaluación Psicológica, 2(22), 91-110.

García, R., y Suárez, R. (2006). Eficacia de un seguimiento a largo plazo con educación interactiva en diabéticos tipo 1. Revista Cubana de Endocrinología, 17(3), (s/p).

Garrido, E. (2000). Autoeficacia en el mundo laboral. Apuntes de Psicología, 18(1), 9-38.

Glasgow, R. E., Ruggiero, L., Eakin, E.G., Dryfoos, J. y Chobanian, L. (1997). Quality of life and associated characteristics in a large national sample of adults with diabetes. Diabetes Care, 20, 562-567.

Gold, J. (2008). Biblioterapia. En P. Aguirán Clemente, L. M. Amurrio López y P. Arranz Carrillo de Albornoz (eds.), Guía de prevención de Burnout para profesionales de Cuidados Paliativos (pp. 57-61). Madrid, España: Arán.

González-Celis, A. L. M., Trón, A. R. y Chávez, B. M.(2009). Evaluación de calidad de vida a través del WHOQOL en población de adultos mayores en México. México: UNAM-FES-Iztacala.

González-Celis, R. A. L. (2009). Autoeficacia para realizar actividades cotidianas (AeRAC) en ancianos mexicanos. En A. L. González-Celis, R. (ed.) Evaluación en Psicogerontología. México: Manual Moderno.

González-Pinto Arrillaga, A. M., Barbeito Resa, S. y Vega Pérez, P. (2010). Abordaje integral del trastorno bipolar. XI Congreso virtual de psiquiatria-Interpsiquis, pp.1-25.

Gordon, P. A. y Benishek, L. A. (1996) The experience of chronic illness: Issues of loss and adjustment. Journal of Personal and Interpersonal Loss, 1(3), 299-307.

Hervás, A., Zabaleta, A., De Miguel, G., Beldarrain, O. y Díez, J. (2007). Calidad de vida relacionada con la salud en pacientes con diabetes mellitus tipo 2. Anales del Sistema Sanitario de Navarra, 30(1), 45-52.

INAPAM, Instituto Nacional de las Personas Mayores, (2016). Ley de los derechos de las personas mayores. (uso público). Instituto Nacional de las Personas Mayores. Recuperado de: https://bit.ly/2nKlKmX [12 de febrero, 2018]

Kliewer, S. y Saultz, J. (2006). Health care and spirituality. United Kingdom: Radcliffe.

Koenig, H. (2001). The healing power of faith. Nueva York, NY: Touchstone.

Landázuri Ortiz, A. M., y Mercado Doménech, S. J. (2004). Algunos factores físicos y psicológicos relacionados con la habilidad interna de la vivienda. Medio ambiente y comportamiento humano, 5(1-2), 89-113.

León, O. G. y Montero, I. (2002). Métodos de Investigación en Psicología y Educación. Madrid, España: McGrawHill.

Levin, J. (2001). God, faith and health. Exploring the spirituality-healing connection. of activity and social support to the functional health of older adults. Journal of Gerontology: Social Sciences, 55, 208-212.

López, F. (2010): ¿Por qué los afectos y la sexualidad en la vejez son asuntos tan importantes?. En L. Felix (ed.), La sexualidad y el afecto en la vejez. Madrid, España: Pirámide.

López, J. (2005): Personas mayores viviendo solas: La autonomía como valor en alza. Madrid, España: Ministerio de Trabajo y Asuntos Sociales.

López, D. M., López, V. M., Ruiz, M. E., López, J. H. y Escobar, A. N. (2004). Disfunción familiar y control del paciente diabético tipo 2. Revista Medica del Seguro Social, 42(4), 281-4.

Losada-Baltar, A. y Montorio-Cerrato, I. (2005). Pasado, presente y futuro de las intervenciones psicoeducativas para cuidadores familiares de personas mayores dependientes. Revista Española de Geriatría y Gerontología, 40, 30-39.

López, G., Orueta S. R., Gómez-Caro, S., Sánchez O. A., Carmona M. J. y Alonso M. F. J. (2009). El rol de Cuidador de personas dependientes y sus repercusiones sobre su Calidad de Vida y su Salud. Revista Clínica de Medicina Familiar, 2(7), 332-339. 
Mata, M., Roset, M., Badía, X., Antoñanzas, F. y Ragel, J. (2003). Impacto de la diabetes mellitus en la calidad de vida de los pacientes tratados en las consultas de atención primaria en España. Atención Primaria, 31, 493499.

Méndez-López, D. M., Gómez-López, V. M. y Navarrete-Escobar, A. (2004). Disfunción familiar y control del paciente diabético tipo 2. Revista Médica del Instituto Mexicano del Seguro Social, 42(4), 281-284.

Menec, V. H. (2003). The relation between everyday activities and successful aging: A 6-year longitudinal study. The Journals of Gerontology Series B: Psychological Sciences and Social Sciences, 58(2), pp.74-82.

Menéndez, M. M. C. y Brochier, K. R. B. (2011). La actividad física y la psicomotricidad en las personas mayores: sus contribuciones para el envejecimiento activo, saludable y satisfactorio. Textos \& Contextos, 10(1), 179192.

Mercado, S. J. (1998). La vivienda: Una perspectiva psicoloìgica. En J. Guevara, A. M. Landaìzuri y A. Teraìn (eds.), Estudios de Psicologiìa Ambiental en Ameirica Latina. Meìxico: BUAP-UNAM-CONACyT, pp. 141-153.

Minuchin, S. (1982). Familias y terapia familiar. Barcelona, España: Gedisa.

Minuchin, S. Nichols, M. P. y Lee, W. (2011). Evaluación de Familias y parejas. Del síntoma al sistema. México: Paidós.

Minuchin, S. y Fishman, H. Ch. (2001). Técnicas de Terapia Familiar. México: Paidós.

Minuchin, S., Rosman, B. 1., Baker, L. (1978). Psychosomatic Families: Anorexia Nervosa in Context. Cambridge, MA: Harvard University.

Molina, C. y Meléndez, J. C. (2007). Análisis cualitativo del bienestar en la vejez. Revista Española de Geriatría y Gerontología, 42, 276-284.

Montorio C. I., Izal F. T. M., López L. A. y Sánchez C. M. (1998). La entrevista de carga del cuidador. Utilidad y validez del concepto de carga. Anales de Psicología, 14(2), 229-248.

Montorio, C. I. (2007). Intervenciones psicológicas en la prevención y atención a la dependencia. Intervención Psicosocial, 16(1), 43-54.

Montorio, I., Díaz-Veiga, P. e Izal, M. (1995). Programas y servicios de apoyo a familiares cuidadores de ancianos dependientes. Geriatria y Gerontología, 3, 157-68.

Muñoz, O., García, P. C. y Durán, L. (eds.) (2004). La salud del adulto mayor. México: Instituto Mexicano del Seguro Social.

Navas, C., Villegas, H., Hurtado, R. y Zapata, D. (2006). La conexión mente-cuerpo-espíritu y su efecto en la promoción de la salud en pacientes oncológicos. Revista Venezolana de Oncología, 18(1), 28-37.

Norris, S. L., Lau, J., Smith, S. J., Schmid, C. H. y Engelgau, M. M. (2002). Self-management education for adults with type 2 diabetes: a meta-analysis of the effect on glycemic control. Diabetes Care, 25, 1159-1171.

Novak, J. y Gowin, D. B. (1988). Aprendiendo a aprender. Barcelona, España: Martínez Roca

Organización Mundial de la Salud. (1984). Aplicaciones en la epidemiología al estudio de los ancianos. Serie de informes técnicos (Núm. 706). Ginebra: OMS.

Organización Mundial de la Salud, División de enfermedades no transmisibles y tecnología de la salud, (1991). Directrices para el desarrollo de un programa nacional para la diabetes mellitus. (Informe OMS/DBO/DM 91-1). Ginebra: OMS

Persini, S. (2011). Valoración desde la perspectiva de los usuarios, un dispositivo de Psicoeducación en diabetes elaborado e implementado en el Centro de Salud No. 10 "Barrio Esperanza" (Tesis de maestría). Recuperado de: http://www.famg.com.ar/documentos/Tesis_sebastian.peresin.pdf

Pinquart, M. y Sörensen, S. (2006). Helping caregivers of persons with dementia: which interventions work and how large are their effects? International Psychogeriatrics, 18(4), 577-95.

Programa Nacional de Diabetes. ISSSTE. Subdirección General Médica. 2007-2011

Reigal, R. E. y Videra, A. (2013). Frecuencia de práctica física y autoeficacia en la tercera edad. Revista Internacional de Medicina y Ciencias de la Actividad Física y del Deporte, 13(49), 107-120.

Rhee, M. K., Cook, C. B., EL-Kebi, I., Lyles, R. H., Dunbar, V. G., Panayioto, R. M., Berkowitz, K. J., Boyd, B., Broussard, S. y George, C. D. (2005). Barriers to diabetes education in urban patients: perceptions, patterns, and associated factors. The Diabetes Educator, 31(3), 410-417.

Rivera-Ledesma, A. y Montero-López, M. (2005). Espiritualidad y religiosidad en adultos mayores. Salud Mental, 28(6), 51-58.

Rivera-Ledesma, A. y Montero-López, M. (2007). Ejercicio clínico y espiritualidad. Anales de Psicología, 23(1), 125-136.

Robles, S. L., Mercado, M. F. J., Ramos, H. I. M., Alcántara, H. E. y Moreno, L. N. C. (1995). Las fuentes de apoyo de los individuos con diabetes en una zona marginal de Guadalajara, Salud Publica, 37(3), 187-196. 
Rubin, R. (2000). Psychotherapy and counselling in diabetes mellitus. En F. Snoek y T. Chas Skinner (eds.), Psychology in Diabetes Care (pp. 235-263). Londres, Reino Unido: Wiley.

Rubio, Llorente. R. y López Vergara. R. (2003). El papel de los familiares y de los profesionales de la salud en la Diabetes, estudio llevado a cabo en el Hospital Ramón y Cajal con la participación del Servicio de Endocrinología y la Facultad de Psicología de la Universidad Autónoma de Madrid. Recuperado de: http:/ /www.diabetes.bayer.es/user/ediarticulos 17.htm

Salvarezza, L. (1998). La Vejez, una mirada gerontológica actual. Buenos Aires, Argentina: Paidós.

Selvini, P. M., Cirillo, S., Selvini, M. y Sorrentino, A. M. (1990). Los juegos psicóticos en la familia. Barcelona, España: Paidós.

Tejada, M., Pastor, M. y Gutiérrez, S. (2006). Efectividad de un programa educativo en el control del enfermo con diabetes. Investigación y Educación en Enfermería, 24(2), 48-53.

Triado, T. C. (2001). Cambio evolutivo, contextos e intervención psicoeducativa en la vejez. Contextos educativos, 4, 119-133.

Valle Coronado Vázquez, López, G. L. M., Martín, R. E., Real, I, J. M., Sánchez, O. M. y Silveira, G. C. (2011). Evaluación en resultados de salud del proceso diabetes tipo 2. Atención primaria, 43(3), 127-133.

Vázquez-Honorato, L. A. y Salazar-Martínez, B. L. (2010). Arquitectura, vejez y calidad de vida. Satisfacción residencial y bienestar social. Journal of Behavior, Health \& Social Issues, 2(2), 57-70.

Zabalegui, Y. A., Navarro, D. M., Cabrera, T. E., Fernández-Puebla, A. G., Bardallo, P. D., Rodríguez, H. E., Gual, G. P., Fernández, C. M., Argemí, R. J. (2008). Eficacia de las intervenciones dirigidas a cuidadores principales de personas dependientes mayores de 65 años. Una revisión sistemática, Revista Española de Geriatría y gerontología, 43(3),157-166. 